\title{
Données préliminaires sur l'exploitation de la malacofaune marine par les groupes ibéromaurusiens de l’abri Alain (Oran, Algérie)
}

First insights into marine malacofauna exploitation by the iberomaurusian groups of the Alain rockshelter (Oran, Algérie)

Emilie Campmas, Amel Chakroun et Souhila Merzoug

\section{OpenEdition}

Journals

Édition électronique

URL : http://journals.openedition.org/paleo/3180

DOI : $10.4000 /$ paleo.3180

ISSN : $2101-0420$

Éditeur

SAMRA

Édition imprimée

Date de publication : 30 décembre 2016

Pagination : 83-104

ISSN : 1145-3370

Référence électronique

Emilie Campmas, Amel Chakroun et Souhila Merzoug, « Données préliminaires sur l'exploitation de la malacofaune marine par les groupes ibéromaurusiens de l'abri Alain (Oran, Algérie) », PALEO [En ligne], 27 | 2016, mis en ligne le 01 juin 2018, consulté le 07 juillet 2020. URL : http://

journals.openedition.org/paleo/3180; DOI : https://doi.org/10.4000/paleo.3180

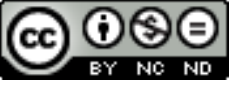

PALEO est mis à disposition selon les termes de la licence Creative Commons Attribution - Pas d'Utilisation Commerciale - Pas de Modification 4.0 International. 


\title{
Données préliminaires sur l'exploitation de la malacofaune marine par les groupes ibéromaurusiens de l'abri Alain (Oran, Algérie)
}

\author{
Emilie CAMPMAS ${ }^{(a)}$, Amel CHAKROUN(b) $^{(b)}$ Souhila MERZOUG(c)
}

\begin{abstract}
Résumé : Si les recherches sur l'lbéromaurusien se sont multipliées ces dix dernières années, les travaux sur les relations Homme-animal restent rares, en particulier sur l'exploitation des ressources marines, alors que de nombreux sites sont actuellement localisés en zone côtière. Le réexamen de la malacofaune marine issue des fouilles de 1930 à l'abri Alain (Oran, Algérie), situé actuellement à cinq kilomètres de la côte méditerranéenne, nous a permis d'obtenir des résultats inédits sur l'acquisition et l'exploitation des ressources côtières par les groupes de l'lbéromaurusien récent ( 15 ka cal. BP). Cette malacofaune a été utilisée à des fins aussi bien alimentaires qu'ornementales. Les mollusques consommés (Patellidae, Mytilidae, Trochidae) proviennent principalement de biotopes rocheux de la zone intertidale, et ont été probablement collectés lors des marées basses. Les lbéromaurusiens ont également ramassé en position secondaire des coquilles de Dentaliidae, Glycymerididae, Cardiidae, Turritellidae pour la confection d'ornements.
\end{abstract}

Mots-clés : Ibéromaurusien, Afrique du Nord, Algérie, malacofaune marine, subsistance, ornements.

Abstract: First insights into marine malacofauna exploitation by the iberomaurusian groups of the Alain rockshelter (Oran, Algérie). Researches regarding Iberomaurusian recently witnessed a significant increase, allowing a better overall understanding of this period. However studies oriented on human-animal relationship are still rare, especially when the exploitation of marine resources is concerned, which is paradoxical as most of the sites are located in coastal areas. In this work, we provide a re-evaluation of the marine malacofauna from the 1930's excavation of the rockshelter Abri Alain (Oran, Algeria), currently located five kilometers from Mediterranean shore. Obtained results document the exploitation of marine resources by recent lberomaurusian population ( $15 \mathrm{ka}$ cal. BP). This malacofauna was used for subsistence but also as ornamentation. The consumed mollusks (Patellidae, Mytilidae, Trochidae) are characteristic of the intertidal zone of rocky shores. These species were probably gathered during low-tides. Shells used as ornamentation (Dentaliidae, Glycymerididae, Cardiidae, Turritellidae) were collected on secondary position.

Key-words: Iberomaurusian, North Africa, Algeria, Marine malacofauna, Subsistence, Ornaments.

\section{Introduction}

Les recherches concernant l'Ibéromaurusien se sont multipliées ces dernières années. Cette culture, qui correspond au Paléolithique supérieur / Later Stone Age en Afrique du Nord, est datée entre 22 et 9,5 ka cal BP environ (e.g., Barton et al. 2007, 2008, 2013, 2016 ; Bouzouggar et al. 2008 ; Linstädter 2008 ; Linstädter et al. 2012 ; Hogue et Barton 2016). Les industries lithiques, caractérisées par la présence de lamelles et de microlithes (Balout 1950 ; Tixier 1963 ; Camps 1974 ; Bouzouggar et al. 2008 ; Linstädter et al. 2012 ; Sari 2008, 2012, 2014 ; Stoetzel et al. 2014 ; Hogue et Barton 2016), semblent évoluer au cours du temps

(a) UMR 5608 TRACES du CNRS, Université Toulouse Jean Jaurès, France - em.campmas@gmail.com

(b) Université de Tunis El Manar, Faculté des Sciences de Tunis, Département de Géologie, El Manar, Tunisie

(c) CNRPAH, Alger, Algérie 
(e.g. Moser 2003 ; Barton et al. 2013). Les recherches récentes croisant les données paléoenvironnementales, chronologiques et culturelles suggèrent que l'lbéromaurusien pourrait se scinder en deux phases majeures, ancienne et récente, s'accompagnant d'un passage de dépôts jaunes à noirs pulvérulents riches en coquilles de mollusques dans les stratigraphies. Selon les publications, cette transition pourrait avoir eu lieu autour de $16 \mathrm{ka}$ cal BP à Ifri n'Ammar (Moser 2003) ou 15 ka cal BP à Taforalt (Barton et al. 2013). II semble également que les données disponibles pour ces deux phases ne soient pas équivalentes, la phase récente étant mieux documentée (Linstädter 2008). Pour cette dernière, l'exemple de Taforalt (Maroc) atteste la présence de nécropoles, où les pratiques funéraires apparaissent complexes (traitement post-mortem des corps, association à des offrandes, notamment de massacres de mouflons à manchettes...) (Mariotti et al. 2009 ; Mariotti, Condemi, Belcastro 2014, 2016 ; Humphrey et al. 2012 ; AoudiaChouarki 2013). Ces populations, qui ont pratiqué l'avulsion des incisives supérieures (Hadjouis 2002 ; Barton et al. 2008 ; Aoudia-Chouarki 2013; Humphrey et Bocaege, 2008 ; De Groote et Humphrey 2015), présentaient des pathologies dentaires (caries) traduisant un mauvais état sanitaire (Poitrat-Targowla 1977 ; Humphrey et al. 2014). L. Humphrey et al. (2014) suggèrent que ces atteintes dentaires pourraient résulter de la consommation de plantes sauvages riches en glucides fermentescibles, ce que les analyses carpologiques semblent confirmer avec la présence de débris de glands de chêne vert et pignons notamment. Ainsi, cette phase récente, qui semble en lien avec des conditions climatiques plus humides, s'accompagnerait de changements comportementaux importants (diversification des ressources exploitées, augmentation du sédentarisme, mise en place de nécropoles, etc.) (Barton et al. 2013).

Cependant, les données sur la subsistance de ces groupes restent rares. Les travaux menés en Algérie sur les faunes vertébrées par S. Merzoug $(2005,2008)$, S. Merzoug et L. Sari (2008) et S. Chibane (2016) suggèrent que les populations ibéromaurusiennes ont consommé les ongulés majoritairement présents dans l'environnement autour des sites (mouflons à manchettes en zones escarpées ou bubales en plaines), tout en intégrant à leur alimentation, en proportion plus faible, une diversité d'autres taxons (lagomorphes, oiseaux, etc.) (Merzoug 2005 ; Chibane 2016). En plus des faunes de vertébrés, la malacofaune terrestre semble occuper une part importante des ressources exploitées (Taylor et al. 2011). Même si de nombreux sites ibéromaurusiens sont localisés en zone côtière, les données sur l'exploitation du milieu marin, et notamment des mollusques marins restent rares, alors qu'ils ont pu être consommés ou utilisés pour la confection d'ornements (Camps 1974 ; Sidi Maamar 1987 ; CampsFabrer 1994).

Dans cet article nous fournissons les premiers éléments issus du réexamen préliminaire de la malacofaune marine des niveaux ibéromaurusiens de l'abri Alain fouillé au début du $20^{\circ}$ siècle par $\mathrm{P}$. Pallary et conservée à l'Institut de Paléontologie Humaine (IPH) de Paris.
Ce travail a plusieurs objectifs concernant la malacofaune marine :

- identifier les espèces exploitées par les Hommes de l'Ibéromaurusien ;

- caractériser le but de leur exploitation : alimentaire et/ou non alimentaire ;

- fournir des données sur les zones et les stratégies d'acquisition ainsi que sur les modalités de traitement ;

- évaluer le potentiel informatif des collections malacologiques anciennes.

\section{1 - L’abri Alain}

L'abri Alain est situé dans la région oranaise (Nord-Ouest de l'Algérie), actuellement à $5 \mathrm{~km}$ de la mer Méditerranée et au nord de la Sebkha d'Oran et à une altitude de $100 \mathrm{~m}$ dans un ravin sur les pentes du Djebel Murdjadjo (fig. 1) (Pallary 1934). La localisation de ce site nous permet donc d'aborder la question du rôle des ressources marines pour les groupes ibéromaurusiens.

L'abri Alain a été découvert par P. Pallary en 1906, qui y a fouillé différents locus de 1927 à 1930 (Pallary 1934). Notons que F. Doumergue, qui a redécouvert ce site en 1914 et l'a fouillé en 1916, l'avait dénommé " grotte des carrières d'Eckmühl » (Doumergue 1935). Cet abri (ou grotte démantelée) est creusé dans le calcaire blanc du Miocène supérieur (étage Sahélien), exploité sous la forme de carrière (Pallary 1934).

Lors de ses fouilles, P. Pallary (1934) décrit une stratigraphie de référence comportant quatre couches, de la plus profonde à la plus superficielle :

- Couche jaune » : $50 \mathrm{~cm}$ d'épaisseur, couleur jaune chrome, compacte, sableuse, pauvre en matériel archéologique et en escargots (principalement Rumina) ; - «Couche jaune-brun »: riche en restes de faune, pulvérulente, sableuse, plus riche en escargots (Archelix et Rumina principalement) et en matériel archéologique, elle comprend aussi de la parure (pétoncles et dentales) ;

- « Couche noire » : pulvérulente, coquilles d'escargots abondantes, coquilles de mollusques marins (patelles, moules, monodontes) en plus des pétoncles et des dentales.

- « Couche brun chocolat » : très dure, riche en galets et pierrailles, présence d'escargots et vertébrés, quelques coquilles de mollusques marins.

Cependant, à l'IPH seules deux couches « jaune » et « noire » sont référencées, comme sur certaines coupes stratigraphiques de P. Pallary (1934) (fig. 2).

D'après H. Hellal (2005) deux datations ont été réalisées par le Centre Scientifique de Monaco en 1975 sur charbon de bois pour la " couche noire". Elles ont fourni les âges de $12550 \pm 190$ BP et $12660 \pm 200$ BP (Hellal 2005), soit entre 15700 et 14100 cal BP (fig. 3).

Deux études du matériel lithique conservé à l'IPH ont été entreprises, l'une par P. Granchon (1987) et l'autre par $\mathrm{H}$. Hellal (2005), respectivement de la « couche jaune » et 

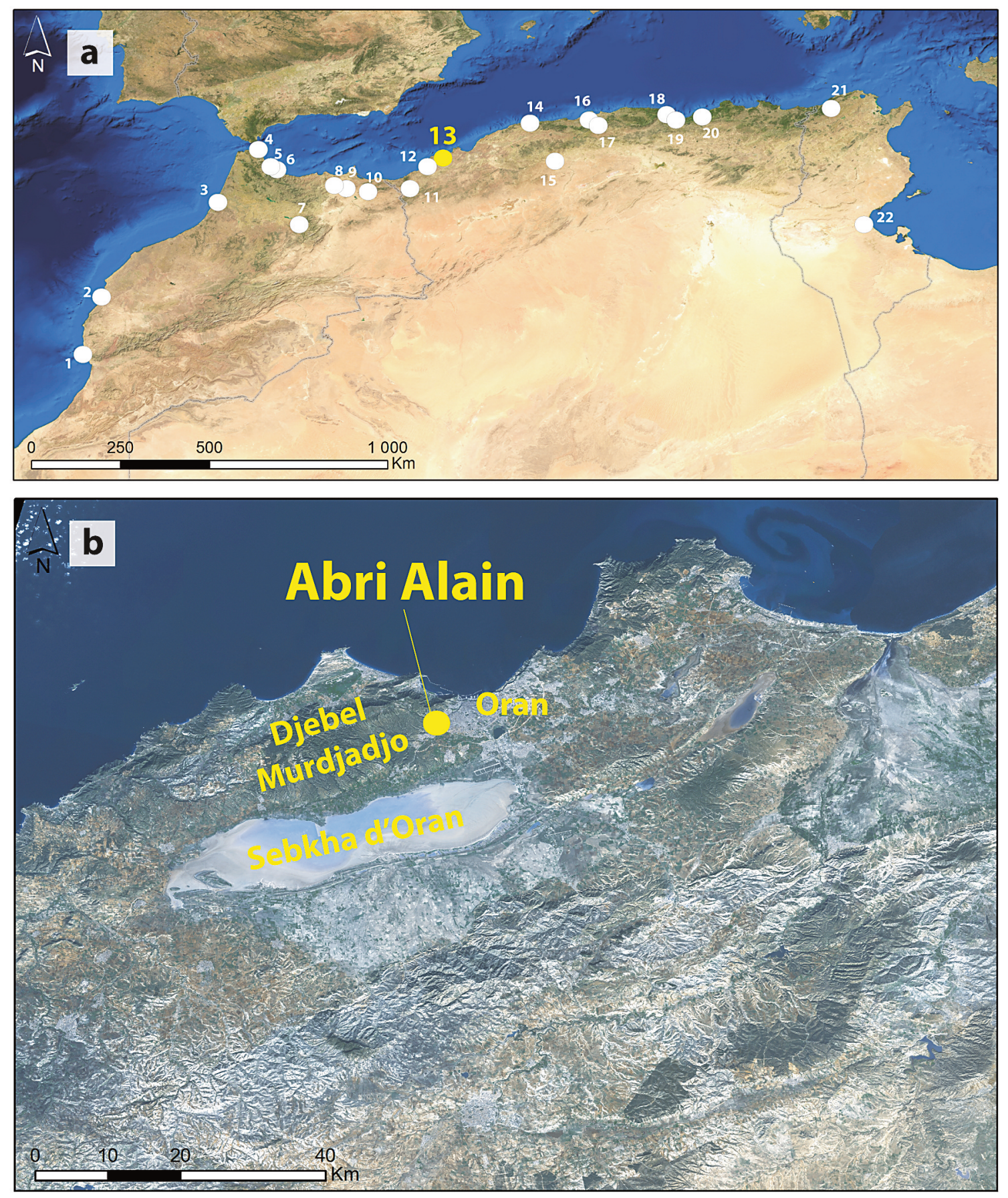

Figure 1 - Localisation de l'abri Alain ; a) parmi les principaux sites ibéromaurusiens [1. Cap Rhir, 2. El Khenzira, 3. Témara, 4. Ghar Cahal, 5. Kehf El Hammar, 6. Hattab II, 7. Kifan Bel Ghomari, 8. Ifri El Baroud, 9. Ifri n'Ammar, 10. Taforalt, 11. La Mouillah, 12. Rachgoun, 13. Abri Alain, 14. Cap Ténes, 15.Columnata, 16. Rolland, 17. Rassel, 18. Afalou Bou Rhummel, 19. Tamar Hat, 20. Taza, 21: Ouchata; 22. Oued-Akarit (adapté de Barton et al. 2013)]; b) dans la région oranaise (Infographie : $M$. Cammas et E. Campmas).

Figure 1 - Location of Alain rockshelter; a) among main iberomaurusian sites [1. Cap Rhir, 2. El Khenzira, 3. Témara, 4. Ghar Cahal, 5. Kehf El Hammar, 6. Hattab II, 7. Kifan Bel Ghomari, 8. Ifri El Baroud, 9. Ifri n'Ammar, 10. Taforalt, 11. La Mouillah, 12. Rachgoun, 13. Abri Alain, 14. Cap Ténes, 15.Columnata, 16. Rolland, 17. Rassel, 18. Afalou Bou Rhummel, 19. Tamar Hat, 20. Taza, 21: Ouchata; 22. Oued-Akarit (adapted from Barton et al. 2013)]; b) in the Oran's region (CAD : M. Cammas et E. Campmas). 
a)

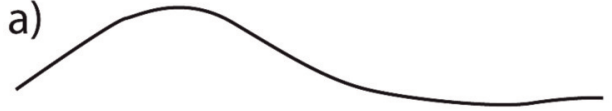

brèche (dôme)

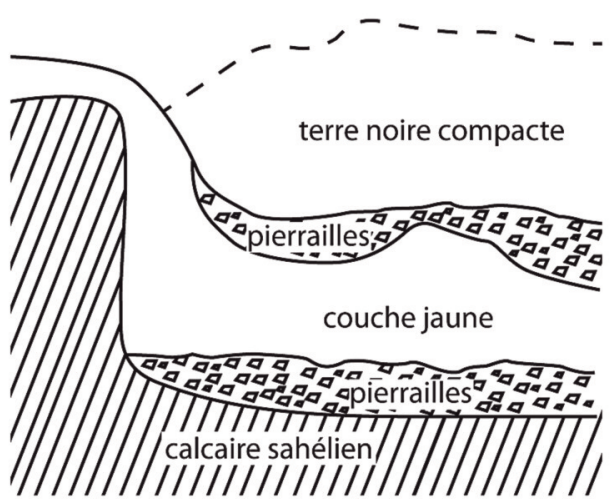

b)

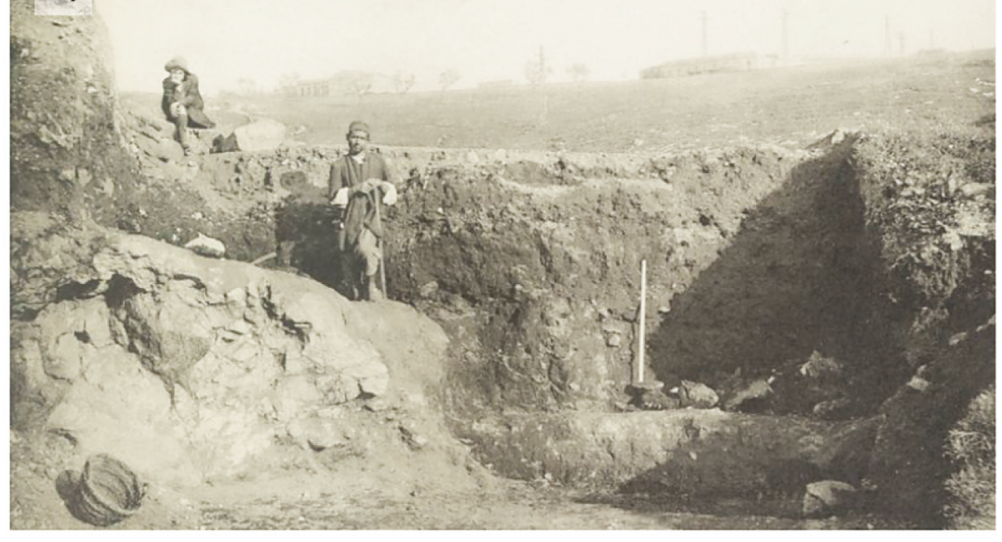

Figure 2 - a) Coupe stratigraphique schématique du remplissage de l'abri Alain du 16 novembre 1927 (d'après Pallary 1934) ; b) Photographie de la tranchée du 25 février 1928 (Photographie G. Buoän dans P. Pallary 1934 - PI. 11).

Figure 2 - a) Schematic stratigraphic section of Alain rockshelter, November 16th, 1927 (from Pallary 1934); b) Photography of the section, February 25th, 1928 (Picture G. Buoän in P. Pallary 1934 - Pl. 11).

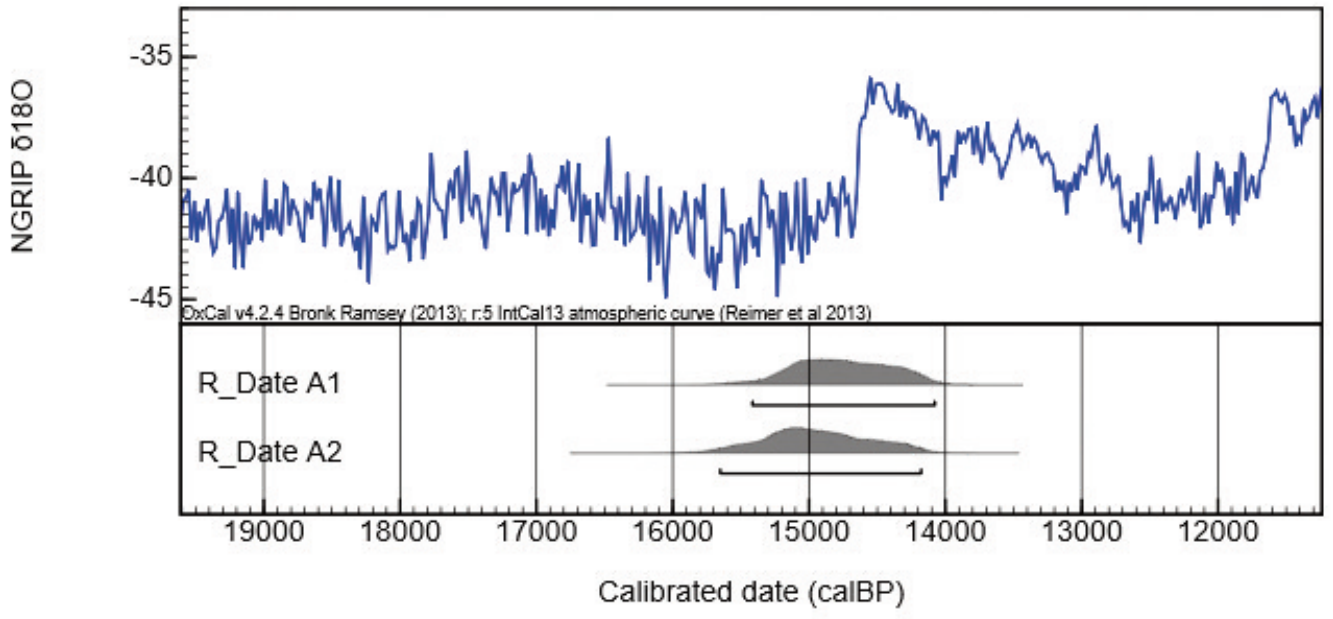

Figure 3 - Ages modélisés de la couche noire de l'abri Alain.

Figure 3 - Modelled ages of the black layer of Alain rockshelter.

de la « couche noire ». P. Granchon (1987) décrit une industrie ibéromaurusienne principalement composée de lamelles à bords abattus, comportant plus rarement d'autres outils tels que des grattoirs ou des burins. H. Hellal (2005) indique quant à lui que l'industrie est caractérisée par un microlithisme accentué. La couche noire comprend elle aussi majoritairement des lamelles à bords abattus associées à de plus rares autres éléments, tels que des grattoirs et des racloirs ou même des microlithes géométriques. Remarquons que si F. Doumergue (1935), en considérant l'industrie lithique et l'absence de céramique, se rallie à l'attribution ibéromaurusienne du dépôt noir, il note cependant des ressemblances avec les dépôts du Néolithique d'autres sites algériens et il émet des réserves quant à l'attribution de la partie supérieure de ce niveau noir.

L'analyse paléontologique de la couche « jaune » (Abbar 2014) et l'étude archéozoologique des couches « noire » et « jaune » (Chibane 2016) suggèrent que parmi les faunes vertébrées, les Hommes ont principalement exploité le mouflon à machettes et le bubale, en parallèle à d'autres taxons moins fréquents, tels que les gazelles, les oiseaux ou les léporidés. 
L'analyse préliminaire de la microfaune des deux couches (observations réalisées par $E$. Stoetzel) a montré l'existence de peu de restes $(<130)$ incluant de la tortue (Testudo graeca), des oiseaux indéterminés, des hérissons (cf. Atelerix algirus) et des rongeurs Gerbillinae (Meriones cf. shawii, Psammomys sp.) qui pourraient témoigner de milieux ouverts semi-arides à arides. Le genre Psammomys ne se retrouve plus actuellement dans la région d'Oran, mais plus au sud dans les zones arides présahariennes. P. Pallary (1934) faisait mention de restes de lézards et de chiroptères, mais ils n'ont pas été observés par E. Stoetzel lors de la révision du matériel. Les plus petites espèces (gerbilles, souris, musaraignes) sont absentes, sans doute en raison de la méthode d'échantillonnage (tamisage grossier à $4 \mathrm{~mm}$ d'après Pallary 1934).

Une première analyse malacologique menée sur les deux couches par H. Sidi Maamar (1987) montre que des mollusques aussi bien marins que terrestres sont présents à l'abri Alain et que si certains (terrestres et marins) ont pu être consommés, d'autres (marins) ont été utilisés comme ornement.

\section{2 - Matériel, intégrité des assemblages et méthode}

\section{1 - Matériel}

Nous avons étudié 1426 restes de mollusques marins (tabl. 1) conservés à l'IPH. Ces éléments semblent, d'après les indications associées au matériel (cartels), provenir des couches « jaune » et « noire ». Néanmoins, tous les vestiges ne sont pas systématiquement associés à des indications de provenance stratigraphique, et aucune information précise de localisation spatiale (plan, locus de fouille, coordonnées) de leur provenance n'a été retrouvée en ce qui concerne les fouilles de P. Pallary (1934).

\section{2 - Intégrité des assemblages}

Si à l'IPH le matériel est attribué à deux couches (« jaune » et " noire »), dans sa description stratigraphique P. Pallary (1934) mentionne quatre couches. De plus, la présence de coquilles de mollusques marins ayant pu être consommés dans des boîtes accompagnées d'étiquettes " couche jaune » interpelle, puisque P. Pallary (1934) précisait que " les seconds [qui ont servi à l'alimentation], ne se trouvent exclusivement qu'à la partie tout à fait supérieure de la couche noire 》(Pallary 1934 - p. 26) et il signale effectivement la présence de «Patelles, Moules, Monodontes " dans la " couche noire » (Pallary 1934 p. 19). En outre, si dans sa description malacologique $P$. Pallary déclare que « Les premiers [qui ont servi d'objet de parure] sont répandus sur toute l'épaisseur du dépôt » (Pallary 1934 - p. 26) ou que « nous voyons apparaître, à la partie supérieure du dépôt, des mollusques marins comestibles (Moules, Patelles, Pourpres, Bigorneaux) et non plus seulement des coquilles d'ornement, comme il y en a tant dans le dépôt jaune et noir » (Pallary 1934 - p. 21), alors que dans sa description stratigraphique il indique leur présence seulement dans les couches « moyenne jaune-brun » et « noire », et ne les mentionne pas dans la couche " jaune inférieure » (Pallary 1934 p. 19). Cependant, lors de son étude, H. Sidi Maamar (1987), qui avait conservé le partitionnement de la malacofaune en " couche noire » et " couche jaune ", décrit des éléments de parures dans ces deux niveaux. Concernant les analyses de la microfaune (observation réalisée par E. Stoetzel) et de la macrofaune (Chibane 2016), elles n'identifient aucune différence taphonomique ou taxinomique entre les niveaux « jaune » et « noir ». À cela s'ajoute la présence de concrétions noires sur la malacofaune analysée par H. Sidi Maamar (1987) et supposée provenir de la couche « jaune ». Dans un but de conservation des collections, le matériel n'a pas été lavé au cours de cette analyse préliminaire, ce qui a limité l'observation des surfaces. II faut noter ici que le sédiment encore présent sur les coquilles des couches « noire » et « jaune » est principalement de couleur noire. Ainsi, au vu de l'histoire complexe de cette collection et des probables mélanges qu'elle a pu subir, nous fournissons ici les résultats de l'analyse des mollusques marins pour l'ensemble de la série, que nous considérons principalement issue de la couche noire, voire de la couche sous-jacente « moyen jaune-brun ». La recherche de remontages inter-couches permettra peut-être de confirmer cette hypothèse.

Par ailleurs, la collection conservée à l'IPH ne semble correspondre qu'à une partie des vestiges excavés. En effet, P. Granchon (1987 - p. 31) indique qu'il est peu probable que le matériel de la dernière année de fouille, 1930, soit inclus dans les collections de l'IPH. Un tamisage avec une maille de $4 \mathrm{~mm}$ avait alors été réalisé (Pallary 1934), ce qui semble être confirmé par l'analyse du matériel lithique qui comprend de très nombreux nucléus et éclats (Granchon 1987). Néanmoins, l'analyse archéozoologique (Chibane 2016) souligne la sous-représentation des petites esquilles osseuses, ainsi que des diaphyses des os longs, portions normalement majoritaires dans les sites anthropiques. La rareté de la microfaune va également dans le sens d'un tamisage réalisé avec une maille importante. II semble donc que les fragments de petite taille soient absents et qu'il ait pu y avoir une sélection différentielle des vestiges à la fouille.

Ainsi, même si l'échantillon observé à l'IPH ne représente probablement qu'une portion de la malacofaune marine présente à l'abri Alain, il nous permet néanmoins de fournir de nouvelles données sur son exploitation par les groupes ibéromaurusiens.

\section{3 - Méthodologie}

La détermination de la malacofaune marine s'est appuyée sur les ouvrages classiques de la malacologie méditerranéenne (e.g. Bucquoy, Dautzenberg, Dollfus 1882-1886, 1887-1889 ; Sacco 1890-1904 ; Locard 1892 ; Perrier 1954). Dans des cas spéciaux se rapportant notamment à la détermination des Patelles, la collection 
personnelle de M. Feki (Département de Géologie de la Faculté des Sciences de Tunis) ainsi que la collection du Muséum National d'Histoire Naturelle - Inventaire National du Patrimoine Naturel (https://inpn.mnhn.fr) ont été consultés.

La détermination des différentes espèces de mollusques et de leurs environnements paléoécologiques s'est aussi basée sur les espèces actuelles référencées en Algérie (Méziane et Kerfouf 2013), ainsi que sur les données malacologiques et paléo-environnementales du Pléistocène supérieur de la Tunisie et du Bassin méditerranéen (Chakroun 2006 ; Chakroun et al. 2009 ; Chakroun et Zaghbib Turki, sous presse). Les dépôts de sédiments recouvrant les surfaces internes et externes des coquilles ont malheureusement limité l'observation et la terminologie descriptive. Nos observations ont été comparées à celles de données antérieures de $\mathrm{P}$. Pallary (1934) et de H. Sidi Maamar (1987). Il semble cependant que nous n'avons pas ré-observé les pièces photographiées par H. Sidi Maamar (1987). Les nomenclatures utilisées par les différents auteurs ont été mises à jour grâce à la base de données : World Register of Marine Species (http://www.marinespecies.org).

La quantification par famille a été réalisée selon la méthode développée par C. Dupont (2006). Ainsi, nous avons décompté le Nombre de Restes (NR) appartenant à des coquillages marins, ainsi que le Nombre Minimum d'Individus (NMI). Pour les gastéropodes tels que les Patellidae, les Trochidae, les Turritellidae, nous avons décompté les NMI à partir des apex. Pour les bivalves, nous avons utilisé les zones avec le crochet. Néanmoins, si pour les Mytilidae nous avons pu différencier les valves droites et gauches, pour les autres bivalves nous avons décompté le nombre de zones avec crochet que nous avons divisé par deux. Pour les scaphopodes tels que les Dentaliidae, dont la coquille est ouverte aux deux extrémités, conique, allongée et plus ou moins courbée (concavité ventrale et convexité dorsale), nous avons utilisé la zone avec une courbure.

\begin{tabular}{|c|c|c|c|c|}
\hline & NR & NMI & $\begin{array}{l}\text { Présence dans } \\
\text { les couches } \\
\text { « noire " (N) et } \\
\text { " jaune " (J) de } \\
\text { l'IPH d'après } \\
\text { les cartels } \\
\text { associés au } \\
\text { matériel }\end{array}$ & $\begin{array}{c}\text { Attribution } \\
\text { couches } \\
\text { « jaune » (J) et } \\
\text { « noire » selon } \\
\text { H. Sidi Maamar } \\
\text { (1987). }\end{array}$ \\
\hline Dentaliidae & 557 & $\sim 270$ & $\mathrm{~J}-\mathrm{N}$ & \\
\hline \begin{tabular}{|l|} 
Glycymerididae cf. \\
Glycymeris nummaria
\end{tabular} & 408 & 109 & $\mathrm{~J}-\mathrm{N}$ & $\mathbb{N}$ \\
\hline $\begin{array}{l}\text { Glycymerididae cf. } \\
\text { Glycymeris pilosa }\end{array}$ & 12 & 6 & $\mathrm{~N}$ & $\mathrm{~N}$ \\
\hline Patellidae & 205 & 140 & $J ?-N$ & $J-N$ \\
\hline $\mid \overline{M y t i l i d a e}$ & 153 & 25 & J?-N & $J-N$ \\
\hline Trochidae & 31 & 12 & J ?-N & $J$ \\
\hline Turritellidae & 21 & 11 & $\mathrm{e}$ & J \\
\hline Cardiidae & 16 & 4 & $\mathrm{~N}$ & $\mathbb{N}$ \\
\hline Muricidae & 2 & 1 & $\mathrm{~N}$ & $\mathrm{~N}$ \\
\hline Ostreidae & 2 & 1 & $\mathrm{~N}$ & $N$ \\
\hline Pectinidae & 1 & 1 & J? & $N$ \\
\hline \begin{tabular}{|l|}
$\begin{array}{l}\text { Empreinte fossile de } \\
\text { bivalves }\end{array}$ \\
\end{tabular} & 17 & & & \\
\hline Serpulidae isolés & 1 & 1 & & \\
\hline Total & 1426 & $\sim 581$ & & \\
\hline Consommés & 391 & 178 & & \\
\hline $\begin{array}{l}\text { Non alimentaires } \\
\text { (ornement) }\end{array}$ & 999 & 397 & & \\
\hline Fonction indet. & 18 & 5 & & \\
\hline
\end{tabular}

Tableau 1 - Spectre en NR et NMI par grandes classes taxinomiques (J?-cartel ne paraissant pas être d'origine).

Table 1 - Malacofaunal spectrum (NR and MNI) by broad taxinomic classes (J?-artefact associated with probably non original label). 
Comme cette collection provient probablement d'une collecte sélective lors des fouilles, nous avons considéré l'état de dégradation des coquilles selon la classification de Dupont (2006) sans la quantifier. En revanche, aucune approche biométrique n'a été menée, limitant la discussion de la sélection des individus selon leur taille.

Même si les coquilles n'ont pas été lavées, nous avons réalisé des observations préliminaires de la surface de ces dernières (à faible grossissement $\times 10$ ) afin de relever d'éventuelles modifications naturelles ou anthropiques. Un échantillon composé de 28 coquilles de différentes espèces a été également observé à la loupe binoculaire à plus fort grossissement (Stéréomicroscope Leica S8 APO, grossissement 10-80x).

\section{3 - Résultats}

\section{1 - Spectre}

Le spectre malacologique comprend de nombreux taxons (tabl. 1).

Concernant les Patellidae, le sédiment encore présent à la surface des coquilles a limité leur identification, cependant nous avons pu ré-observer les espèces décrites par Pallary (1934). Nous avons identifié principalement des Patella ferruginea et des Patella caerulea. Patella ferruginea, généralement de grande taille $(50-70 \mathrm{~mm})$, a été facilement identifiable, même à l'état fragmentaire, grâce à ses côtes puissantes et prononcées (Perrier 1954). Nous avons reconnu Patella caerulea par la forme de sa coquille robuste, assez aplanie, de forme conique, avec un sommet légèrement antérieur et une base arrondie denticulée, dont la face externe est composée de nombreux sillons rayonnants inégaux légèrement ondulés et de fines stries d'accroissement concentriques (Bucquoy et al. 1882-1886 ; Rampal 1965). Parmi les différents spécimens de Patella caerulea nous avons distingué la forme Patella caerulea typique et la variété subplana de Potiez et Michaud (1838). La coquille de cette dernière est pentagonale à côtes rayonnantes renflées et à sillons également rayonnants. Son péristome est arrondi, tranchant, irrégulièrement plissé et denticulé, avec des stries d'accroissement nombreuses et rapprochées donnant à la surface un aspect légèrement rugueux (fig. 5b). Ces observations correspondent parfaitement à la diagnose de D.E. Bucquoy et al. (18821886) et J. Rampal (1965). Nous avons également repéré un autre spécimen avec des affinités de Patella caerulea variété subplana mutation stellata (Bucquoy et al. 18821886 ; Rampal 1965). Elle se distingue par des côtes rayonnantes qui déterminent des prolongements anguleux donnant à la coquille un aspect étoilé (fig. 5a). Cependant, aujourd'hui, toutes ces formes sont regroupées sous l'appellation Patella caerulea. De plus, un individu (en état fragmentaire) pourrait également appartenir à Patella rustica qui se singularise par sa surface granuleuse, parsemée de ponctuations noires et sa hauteur généralement plus élevée que Patella caerulea (Bucquoy et al. 1882-1886). Notons que H. Sidi Maamar (1987) avait identifié l'espèce Patella aspersa, qui n'a été repérée ni par
P. Pallary (1934) ni dans la collection de l'IPH que nous avons observée. Patella aspera est caractérisée par une coquille conique à base ovale denticulée et faiblement rétrécie antérieurement, avec un sommet peu déporté vers l'avant. Elle est également ornée de nombreuses côtes rayonnantes inégales et rugueuses coupées par des stries d'accroissement concentriques qui déterminent des aspérités épineuses (Bucquoy et al. 1882-1886 ; Rampal 1965). Dans notre cas, le sédiment recouvrant les coquilles limite l'identification des caractères morphologiques nécessaires pour la distinction entre Patella caerulea et Patella aspera, qui en plus, selon G. Sella, Robotti, Biglione (1993), ont des colorations et des morphologies similaires, qui les rendent parfois difficiles à distinguer.

Parmi les Trochidae, nous avons repéré Phorcus turbinatus qui est aisément identifiable par son ouverture arrondie, sa coquille solide et épaisse, légèrement élevée et composée de six tours convexes, dont le dernier est régulièrement bombé, avec la région ombilicale légèrement calleuse (Bucquoy et al. 1882-1886 ; Locard 1892). Certains des spécimens observés présentent encore une pigmentation de coloration grise marquée de taches rougeâtres parfois violacées, subquadrangulaires et allongées (fig. 5l).

Nous avons également observé de rares fragments de coquilles de Stramonita haemastoma, gastéropode courant dans les dépôts pléistocènes supérieurs méditerranéens (Chakroun, Zaghbib-Turki, Moncef-Turki 2016 ; Chakroun et Zaghbib-Turki sous presse). Cette espèce d'assez grande taille $(50-60 \mathrm{~mm})$ est ornée de tubercules et est caractérisée par sa dernière spire développée et son ouverture large (Perrier 1954).

Les Turritellidae appartiennent à l'espèce Turritella triplicata qui est un gastéropode présentant une coquille à ouverture arrondie, nombreuses spires, lui octroyant une forme allongée (Hörnes 1852-1856 ; Esu et Girotti 2010).

Les coquilles de Scaphopodes sont nombreuses et sur le plan taxinomique correspondent principalement à la famille des Dentaliidae. Les fragments que nous avons pu identifier semblent appartenir à trois espèces communément rencontrées en Méditerranée et décrites par P. Pallary (1934) : Antalis vulgaris, Antalis inaequicostata et Fustaria rubescens.

Les Cardiidae sont représentés par Cerastoderma edule dont la coquille est caractérisée par des côtes rayonnantes marquées, croisées par des stries d'accroissement bien visibles sans épines ni tubercules saillants (Bucquoy et al. 1987-1998) et Acanthocardia tuberculata qui a une coquille épaisse avec des côtes marquées, robustes et rayonnantes présentant des épines courtes, parfois noduleuses ou absentes (Perrier 1954).

Les coquilles de Glycymerididae observées sont érodées. L'érosion de la face externe se manifeste par l'érosion des lignes de croissance et celle de la face interne par l'usure des dents du plateau cardinal et des crénelures se trouvant sur le bord palléal des coquilles. Par conséquent, la détermination spécifique est extrêmement délicate. Deux 
espèces semblent pourtant être présentes : Glycymeris pilosa et Glycymeris nummaria d'après P. Pallary (1934).

Parmi les Pectinidae, H. Sidi Maamar (1987) avait distingué l'espèce Pecten maximus cependant, les fragments qu'il présente semblent correspondre à la valve dorsale de Pecten jacobaeus qui concorde avec la diagnose M. Feki (1975) (convexité plus ou moins forte de la valve et section arrondie des côtes).

Concernant les Ostreidae, P. Pallary (1934) avait identifié Ostrea edulis. H. Sidi Maamar (1987) indique la présence de Crassostrea angulata mais il s'agit probablement d'une confusion avec Ostrea edulis qu'il considère comme synonyme, car Crassostrea angulata est une espèce importée d'Asie en Europe au $19^{\circ}$ siècle (Huvet 2000 ; Pascal, Clergeau, Lorvelec 2010).

Les coquilles des tubiformes sont extrêmement difficiles à déterminer. Ces dernières correspondent soit à des
Annélides de la famille des Serpulidae soit à des mollusques de la famille des Vermetidae.

P. Pallary (1934) et H. Sidi Maamar (1987) avaient également mentionné la présence de Ranellidae (Charonia lampa) et de Cerithiidae (Cerithium vulgatum), taxons que nous n'avons pas re-observés, mais qui semblent communs dans les associations de la faune quaternaire marine de l'Afrique du Nord (Chakroun 2006 ; Chakroun et al. 2009 ; Chakroun, Zaghbib-Turki, Moncef-Turki 2016).

Notons également la présence de vertèbres de poisson et de fragments de tests d'oursin. Une dent fossile, pouvant appartenir à un mégalodon (fig. 4c), avait déjà été identifiée par P. Pallary (1934). Des empreintes fossiles de coquilles de mollusques (fig. $4 a$, b) sont également présentes. Ces dernières proviennent très certainement de l'encaissant qui est un calcaire du Miocène supérieur (Sahélien) blanc zoogène marin à intercalation de marnes riches en restes de poisson et en coquilles de mollusques (Gentil 1903 ; Roger 1942 ; Freneix, Saint-Martin, Moissette 1987) ou de
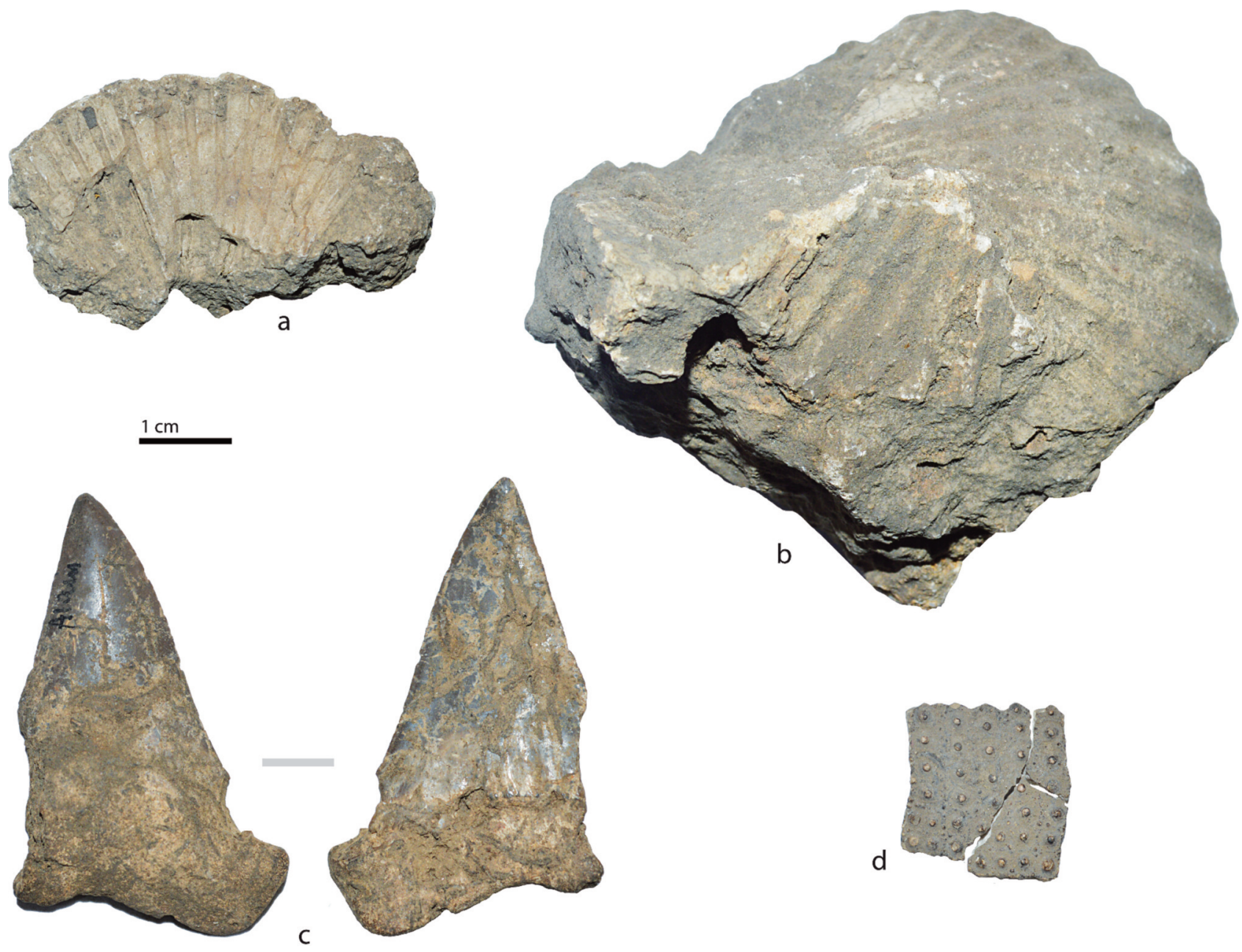

Figure 4 - a-b) Empreintes de coquilles de mollusques fossiles ; c) dent de mégalodon ; d) test d'oursin (Photos : E. Campmas).

Figure 4 - a-b) Fossil shell impressions; c) megalodon tooth; d) sea urchin shell (Pictures E. Campmas). 
dépôts proches, puisque les marnes à silex qui constituent le sahélien du Djebel Murdjadjo renferment de nombreux restes de poissons fossiles et notamment Carcharodon megalodon (Arambourg 1927).

\section{2 - Quelles espèces consommées ?}

L'observation macroscopique des surfaces des rares Patelles nettoyées suggère qu'elles n'ont pas subi une longue exposition sur la plage avant d'avoir été collectées (fig. 5a). II semble en être de même pour les Mytilidae. Certaines coquilles de Patelles présentent des encoches (fig. 5b) rappelant celles laissées lors de leur collecte actuelle à l'aide de couteaux glissés entre la coquille et le rocher pour faire levier (fig. 5c ou Nouet et al. 2015). D'autres coquilles présentent une fragmentation plus importante, à différents stades de dégradation suivant la typologie proposée par C. Dupont (2006) (fig. 5d-f). Les coquilles des Patelles ayant tendance à se fragmenter selon des zones de fragilité préférentielle, correspondant aux lignes circulaires de croissance (e.g., Dupont 2006), la fragmentation observée peut être associée à un processus taphonomique post-dépositionnel tel que le piétinement ou la compaction des sédiments. Une valve de moule complète arbore également une encoche dans une zone peu fragile de la coquille (fig. 5h). Cette encoche rappelle là aussi celles pouvant être produites actuellement lors de l'ouverture des moules sur le littoral méditerranéen par les commerçants de mollusque de Port de Grau de Vendres (Hérault) en vue de leur consommation crue (observation personnelle E. Campmas) (fig. 5i-j). L'absence de l'apex sur certaines coquilles de Trochidae (fig. 5I) pourrait s'expliquer par le retrait de cette partie pour faciliter l'extraction de la chair (Russell et al. 1995 ; Bosch et al. 2015).Les zones de plus grande fragilité, zones préférentielles de fragmentation des Trochidae, correspondent aux sutures des tours de spires (e.g. Dupont 2006).

Pour les coquilles de Patellidae, Mytilidae et Trochidae, l'absence de traces de bioérosion par des lithophages, ainsi que d'érosion mécanique liée au ressac des vagues, vont dans le sens de la collecte de ces mollusques vivants. Les traces compatibles avec la collecte ou l'extraction intentionnelle (encoches sur les coquilles de Patellidae et Mytilidae et absence de la zone de l'apex sur les coquilles de Trochidae) pourraient confirmer la collecte de ces mollusques frais dans un but alimentaire. Au vu de l'état fragmentaire et du très faible nombre de restes identifiés pour les Muricidae (fig. $5 \mathrm{~m}$ ), les Ostreidae, les Cardiidae (Cerastoderma edule), voire même les Pectinidae, leur participation à l'alimentation ne peut être ni rejetée ni assurée. Les tubiformes ont probablement été ramenés involontairement (fig. 5n), accrochées sur la coquille d'autres mollusques (fig. 5h).

Les Pectinidae et les Cerastoderma edule vivent dans la zone infra-tidale, parfois enfouis dans le substrat sableux, mais leur participation à l'alimentation n'est pas certaine ici. Les mollusques dont la consommation est assurée (Mytilidae, Patellidae, Trochidae) se rencontrent dans un biotope rocheux de la zone intertidale, où ils ont probablement été collectés lors des marées basses. Selon leur sensibilité à la dessiccation, les différentes espèces de Patellidae n'évoluent pas dans les mêmes parties de la zone intertidale. Patella ferruginea se rencontre plutôt dans la partie supérieure de la zone intertidale, alors que Patella caerulea et Patella aspera sont plutôt inféodées à la partie inférieure de la zone médio-littorale et infra-littorale, dans les zones à humification permanente, bien que Patella aspera pourrait se rencontrer dans des zones légèrement plus profondes que Patella caerulea. La différence principale de répartition entre ces deux dernières espèces réside plutôt dans la variabilité d'exposition aux vagues. En effet, Patella aspera semble se rencontrer plutôt sur les substrats rocheux soumis à une forte action des vagues, contrairement à Patella caerulea (Rampal 1965 ; Sella et al. 1993). Ainsi, tout en gardant en tête la difficulté de différenciation de Patella caerulea et Patella aspera, en particulier dans le cas de cette collection non nettoyée, nous pouvons émettre l'hypothèse que même si les chasseurs-collecteurs de l'lbéromaurusien de l'abri Alain semblent avoir exploité tous les niveaux de l'estran, une réelle absence de Patella aspera qui affectionne les environnements profonds et agités, pourrait indiquer que les Hommes ont évité certaines zones pouvant être dangereuses.

Si les Mytilidae qui vivent en agrégats peuvent être collectés en masse par section du byssus, les Patellidae, plus délicates à décrocher du substrat rocheux, doivent être collectées une par une (e.g. Fa 2008). Les encoches sur les coquilles de Patellidae pourraient aller dans le sens de leur collecte avec un outil adapté, par exemple un outil plat glissé entre la coquille et le rocher. Les patelles peuvent être également collectées sans outils, par exemple la nuit lorsqu'elles se nourrissent ou par simple percussion, avec un galet (e.g. Russell, Bonsall, Sutherland 1995). Les Mytilidae sont représentés par des individus de différentes tailles, dont quelques-uns de faible dimension (juvéniles) (fig. $5 \mathrm{~g}$ ) indiquent qu'aucune sélection des individus les plus grands n'aurait été opérée lors de la collecte. En effet, même dans le cas où les moules de grande dimension sont recherchées, la collecte en masse de moules peut engendrer la récolte de différentes catégories des tailles de coquilles, le tri pourra alors être réalisé après collecte (observation personnelle réalisée par E. Campmas dans le cadre du projet ACoAPass - fonds d'amorçage TRACES).

Concernant les modalités de traitement, si la chauffe des moules peut favoriser leur ouverture elle ne laissera pas de traces dans le cas de l'utilisation d'un contenant au contraire d'une cuisson directe sans contenant sur ou sous la braise (e.g. Aldeias et al. 2016). Seule une étude attentive de l'état des surfaces des coquilles et des analyses physico-chimiques (FTIR) permettraient peut-être d'apporter des éléments de réponse à cette réflexion. L'encoche observée sur l'une des coquilles de moule indiquerait plutôt leur consommation crue, de la même façon que le retrait de l'apex pour les Trochidae.

En parallèle à la consommation des mollusques, les Hommes ont dû également consommer des oursins 
a
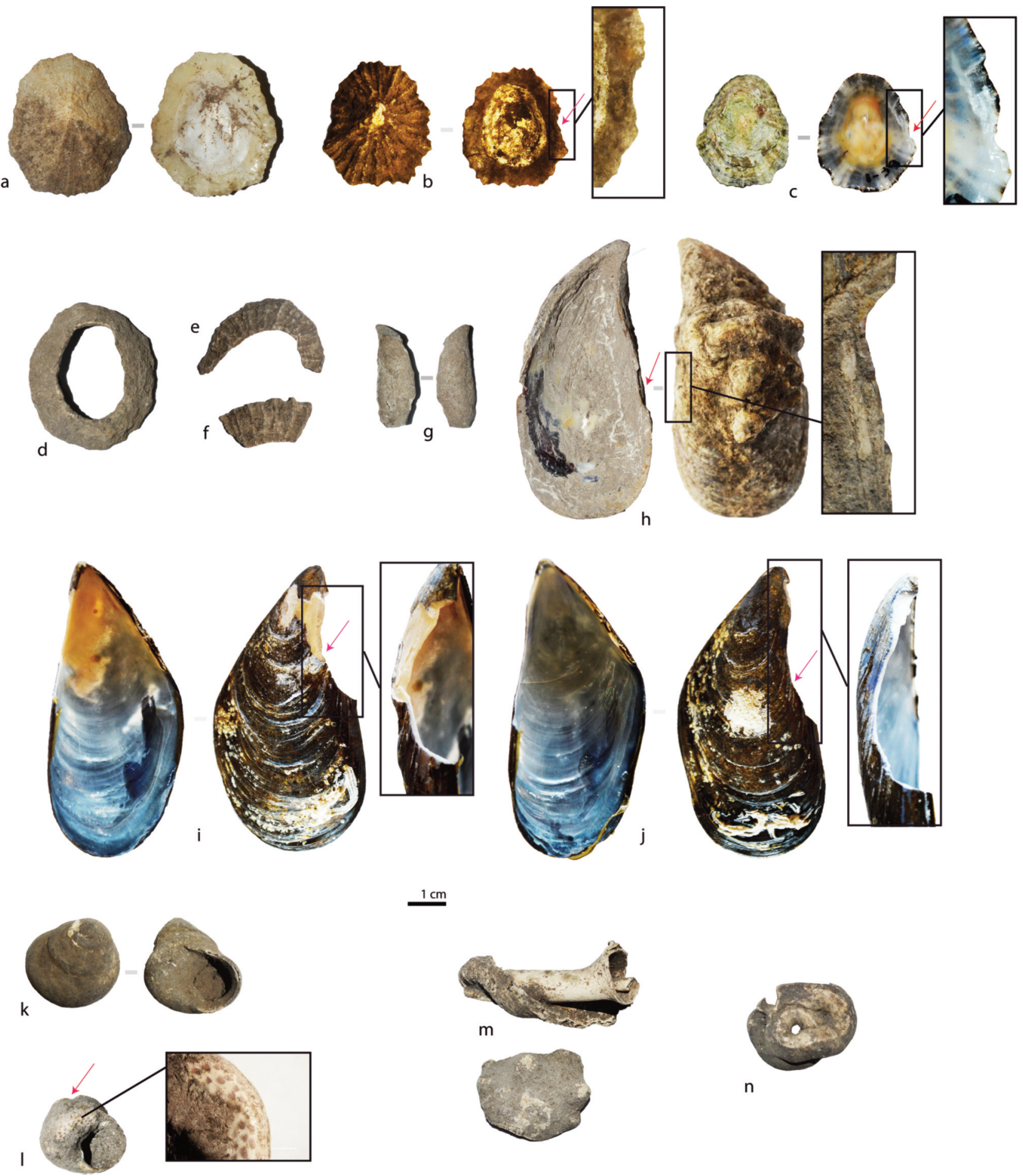

$\mathrm{n}$

Figure 5 - Coquilles de mollusques consommés et ramenés accidentellement à l'abri Alain et comparaison avec des observations actuelles ; $a-f)$ Patellidae [ $a-b, d-f$ ) Patellidae de l'abri Alain (avec a-b) Patella caerulea) ; c) Patellidae actuels] [c) flèches : encoches ; dégradation des coquilles d'après la typologie de C. Dupont (2006) : a-b) coquilles entières, d) anneau de coquille, e) fragment de coquilles en forme de croissant égal à la moitié d'un anneau, f) fragment de coquille en forme de croissant inférieur à plus de la moitié de l'anneau] ;

$g$-j) Mytilidae[h-j) Mytilus galloprovincialis, $g$-h) Mytilidae de l'abri Alain, $i-j)$ Mytilidae actuels] [h-j) flèche : encoches]; $k$-l) Trochidae (Phorcus turbinatus)de l'abri Alain [l) flèche : absence de la zone de l'apex] ; $m$ ) fragments de coquilles de Muricidae (Stramonita haemastoma) de l'abri Alain ; n) Tubiforme de l'abri Alain (Photos E. Campmas).

Figure 5 - Shells of consumed and accidentally introduced mollusks at Alain rockshelter and comparison with modern observations; a-f) Patellidae [a-b, $d-f$ ) Patellidae from Alain rockshelter (with $a-b)$ Patella caerulea; $c$ ) modern Patellidae] [c) arrows: notches; fragmentation of the shell according to $C$. Dupont (2006) typology: $a-b$ ) complet shell, d) ring of shell, e) crescent shaped fragment of shell equal to a half ring, f) crescent shaped fragment of shell smaller than a half ring]; $g-j)$ Mytilidae [h-j: Mytilus galloprovincialis, $g$-h) Mytilidae from Alain rockshelter, i-j) modern Mytilidae] [h-j) arrows: notches]; $k$-l) Trochidae (Phorcus turbinatus) from Alain rockshelter [l) arrow: lack of apex]; $m$ ) shell fragments of Muricidae (Stramonita haemastoma) from Alain rockshelter; $n$ ) Tubiforme from Alain rockshelter (Pictures E. Campmas). 
(présence de tests) (fig. 4d) et peut-être du poisson (présence de vertèbres et de mandibules) (Chibane 2016).

\section{3 - Ressources non alimentaires}

L'utilisation de certaines des coquilles en parure (Glycymerididae et dentales) avait déjà été relevée par P. Pallary (1934) et H. Sidi Maamar (1987).

De nombreux fragments de Scaphopodes sont recensés (fig. 6). Ces derniers présentent différentes teintes (fig. 6a) et certains portent encore des résidus de pigments (fig. $6 \mathrm{~b}$ et d2). Des fragments de Scaphopodes de grande taille sont émoussés, notamment dans les zones d'ouverture et peuvent même présenter des stries liées à de l'abrasion (fig. 6e). Deux Scaphopodes sont encore emboîtés, maintenus par du sédiment concrétionné (fig. 6c).

Les valves de Glycymerididae cf Glycymeris nummaria complètement émoussées (après fragmentation) sont nombreuses (fig. 7a-g, j-l et n-p). Parmi elles, celles avec des perforations sont abondantes (fig. 7a-g; $\sim 25 \%$ des zones avec crochet). Ces dernières peuvent également être cassées ou ouvertes (fig. 7j-I ; 30\% des zones avec crochet). Certaines perforations peuvent avoir une origine naturelle (fig. $7 \mathrm{~m}$ et q), qui peut être générée par l'érosion des parties convexes (crochet, bord de la coquille, dents de la charnière) sur le sable par le ressac des vagues ou par l'action de mollusques carnivores, comme par exemple certains Naticidae ou Muricidae, qui avec leur radula percent les coquilles de mollusques afin de se nourrir des parties moles (Loppens 1926 ; Dupont 2006, 2014 ; Cabral et Martin 2016). Cependant, certaines perforations présentant des traces (stries, enfoncements) (fig. $7 \mathrm{v}$ et w) évoquent la possibilité de perforations volontaires (par raclage et/ou percussion légère). De rares résidus de pigments ont été identifiés sur des coquilles observées à fort grossissement. Quelques spécimens de Glycymerididae cf. Glycymeris pilosa $(\mathrm{N}=12)$, d'une épaisseur de coquille plus réduite (fig. $7 \mathrm{~h}$ et $\mathrm{u}$ ), ne présentent pas la même patine et sont moins émoussés. Si comme pour les Glycymerididae cf. Glycymeris nummaria certaines coquilles présentent des perforations dans la zone du crochet, en revanche toutes les valves sont complètes.

Des Cardiidae présentent un aspect moins patiné que les Glycymerididae of Glycymeris nummaria, mais l'un d'entre eux (Acanthocardia tuberculata) arbore également une perforation dans la zone du crochet (fig. 7i).

Certaines coquilles de Turritellidae présentent des perforations (fig. 8). L'une des coquilles perforées arbore des usures aussi bien sur les bords de la perforation qu'autour du péristome. Des résidus de pigment ont également été observés sur cette coquille.

Les Scaphopodes évoluant quasi exclusivement en eau profonde (Lindner 2015), les coquilles ont dû être ramassées en position secondaire, une fois rejetées sur les plages ou même en position fossile. L'état d'érosion des coquilles de Glycymerididae cf. Glycymeris nummaria suggère qu'elles ont été collectées longtemps après la mort des mollusques, roulées sur la plage ou plus probablement en position fossile. Ainsi, la présence des émoussés, de perforations naturelles, ou d'animaux loin de leur environnement naturel (eaux profondes) indique que ce n'est pas par intérêt alimentaire que les Hommes ont ramassé les Dentaliidae, Glycymerididae, Cardiidae (Acanthocardia tuberculata) et Turritellidae. P. Pallary (1934) avait déjà indiqué qu'une partie de ces fossiles pourrait provenir des calcaires sahéliens du Miocène (qui comprend de nombreuses coquilles de bivalves dont des Pectinidae et des Ostreidae) (Gentil 1903 ; Roger 1942 ; Freneix, Saint-Martin, Moissette 1987) ou des plages soulevées du Pliocène supérieur et du Pléistocène de la région d'Oran. L'acquisition de matériaux en position fossile s'envisage également pour la dent de mégalodon (fig. 4c) ou les empreintes de bivalves fossiles (fig. 4a, b). En effet, P. Pallary (1934 - p. 43) suggérait déjà que « nous avons trouvé plusieurs empreintes sur calcaire sahélien qui ont été exactement découpées en suivant le contour des valves. II s'agit donc bien d'objets collectés à dessein ". Cependant concernant les scaphopodes. P. Pallary (1934 - p. 44) indiquait qu' " ils manquent dans les plages soulevées, mais on en trouve dans les gisements pliocènes, où ils sont d'ailleurs rares » et il proposait une origine plus lointaine pour ces derniers.

Certaines perforations, usures et résidus de pigments attestent de l'utilisation de ces éléments probablement comme ornements.

Concernant les proportions, dans notre cas, les coquilles de mollusques utilisées à des fins non alimentaires, en particulier les Scaphopodes, sont mieux représentées que celles appartenant aux mollusques consommés. Ces fréquences sont similaires aux observations effectuées par P. Pallary (1934) « les plus nombreux sont les dentales... puis viennent les pétoncles » (Pallary 1934 - p. 42). Cependant, les fouilles ayant eu lieu dans les années 1930, il est probable que les archéologues de l'époque aient pris soin de conserver préférentiellement les vestiges utilisés pour l'ornementation.

\section{4 - Discussion}

\section{1 - Exploitation des mollusques marins à l'lbéromaurusien}

D'après H. Camps-Fabrer (1994) les Patellidae sont répandus dans les sites ibéromaurusiens de la façade méditerranéenne. Par exemple, à la grotte Rassel (Algérie, fig. 1-17), C. Brahimi (1970) note la présence de Trochidae et de Patellidae. Ces familles sont également bien représentées dans la région des Babors (Afalou Bou Rhummel, Tamar Hat et Taza 1 ; fig. 1-18-19-20) (Arambourg et al. 1934 ; Saxon et al. 1974 ; Merzoug accepté). À Rachgoun (Algérie, fig. 1-12), H. Camps-Fabrer (1994) indique qu'en plus des coquilles de Mytilidae, Patellidae, Trochidae, des spicules d'oursin sont présents. Cependant, elle note l'absence de restes de poisson, ce qui n'est pas le cas à l'abri Alain ou dans les sites de la région 

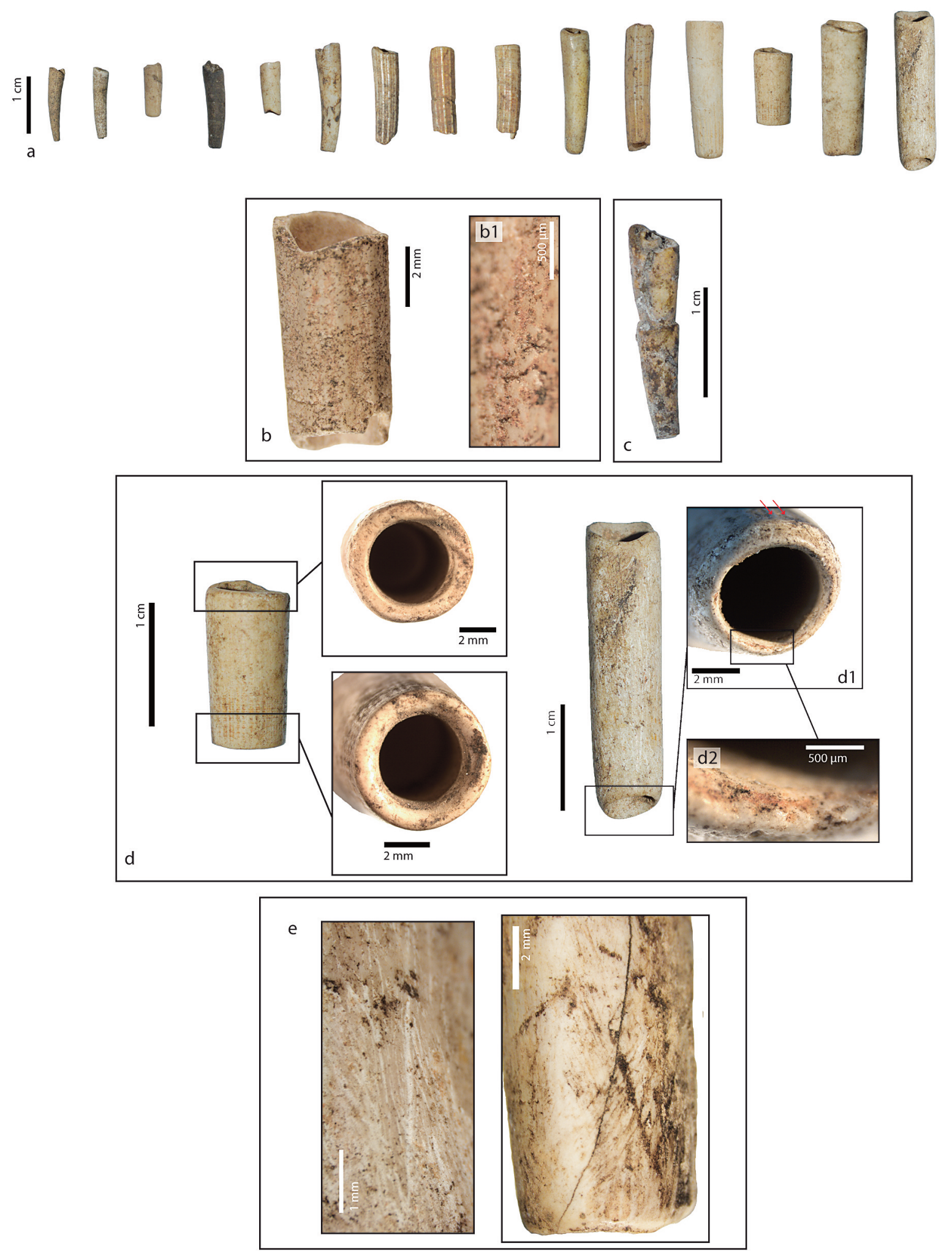

Figure 6 - Coquilles de scaphopodes de l'abri Alain ; a) exemples des aspects de surface et des teintes ; b) avec des résidus de pigments ; c) coquilles encore emboîtées ; d) fragments avec des émoussés dans les zones d'ouverture [il est à noter que le spécimen de droite, entièrement travaillé, est le seul specimen difficile à déterminer, mais il a été rapproché d'un scaphopode du fait de la présence de deux côtes d'ornementation longitudinales encore légèrement visibles (flèches en d1)] ;d2 : avec des résidus de pigments; e) stries d'abrasion (Photos E. Campmas).

Figure 6 - Shells of Scaphopodia from Alain rockshelter; a) examples of surface aspects and colorations; b) with pigment residues; $c$ ) shells still nested; d) fragments with smooth opening [note that the worked right specimen is the only one difficult to identify, but the presence of tow slight longitudinal ornamental ribs (arrows in d1) suggests its attribution to scaphopodia]; d2: with pigment residues; e) abrasion marks (Pictures E. Campmas). 

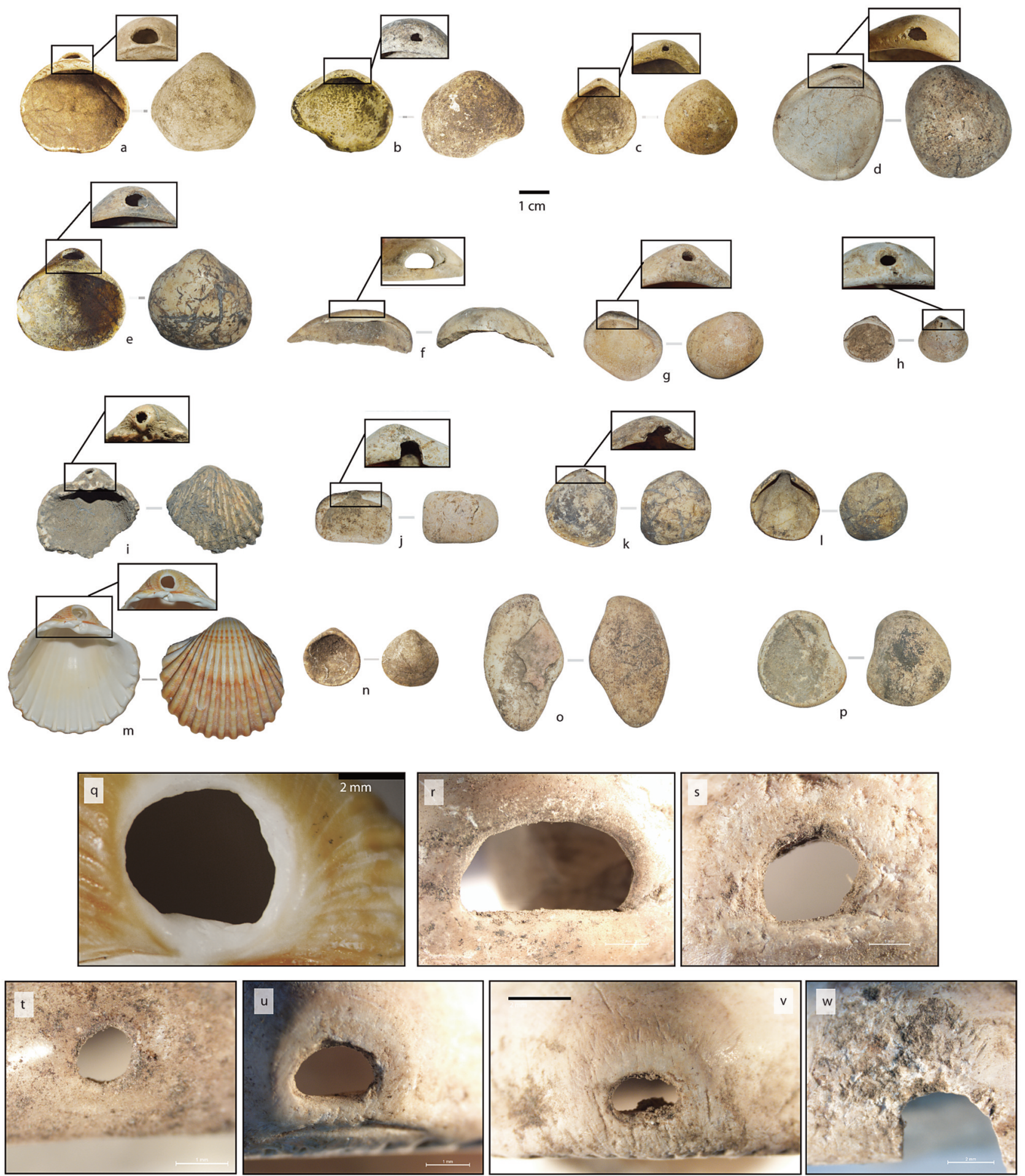

Figure 7 - Coquilles de bivalves n'ayant pas servi à des fins alimentaires ; a-l, $n-p, r$-w) bivalves de l'abri Alain, $m$, q) bivalve actuel ramassé sur la plage de Skhirat (Maroc) [a-g, $j-1 ; n-p, r$-w) Glycymerididae cf Glycymeris nummaria, $h$ ) Glycymerididae cf. Glycymeris pilosa ; $j, m)$ Cardiidae (Acanthocardia tuberculata), a-i, $m$ ) perforés, $k-l)$ perforation « ouverte », n) non perforé, o-p) fragments non perforés, $q-w)$ observation des perforations à fort grossissement : q) perforation naturelle actuelle, v) perforation associée à des stries, w) perforation " ouverte " associée à des enfoncements (Photos E. Campmas).

Figure 7 - Shells of non consumed bivalves; a-l, $n-p, r$-w) bivalves from Alain rockshelter, $m, q)$ modern bivalves collected on the Skhirat beach (Morocco) [a-g, j-l; n-p, r-w) Glycymerididae cf Glycymeris nummaria, h) Glycymerididae cf. Glycymeris pilosa; j, m) Cardiidae (Acanthocardia tuberculata), $a-i, m$ ) with perforation, $k-I)$ " open » perforation, $n$ ) without perforation, $o-p$ ) fragments without perforation, $q-w)$ observation of perforations at high magnification: q) modern natural perforation, v) perforation associated with streak-marks, w) "open" perforation associated with punctures (Pictures E. Campmas). 

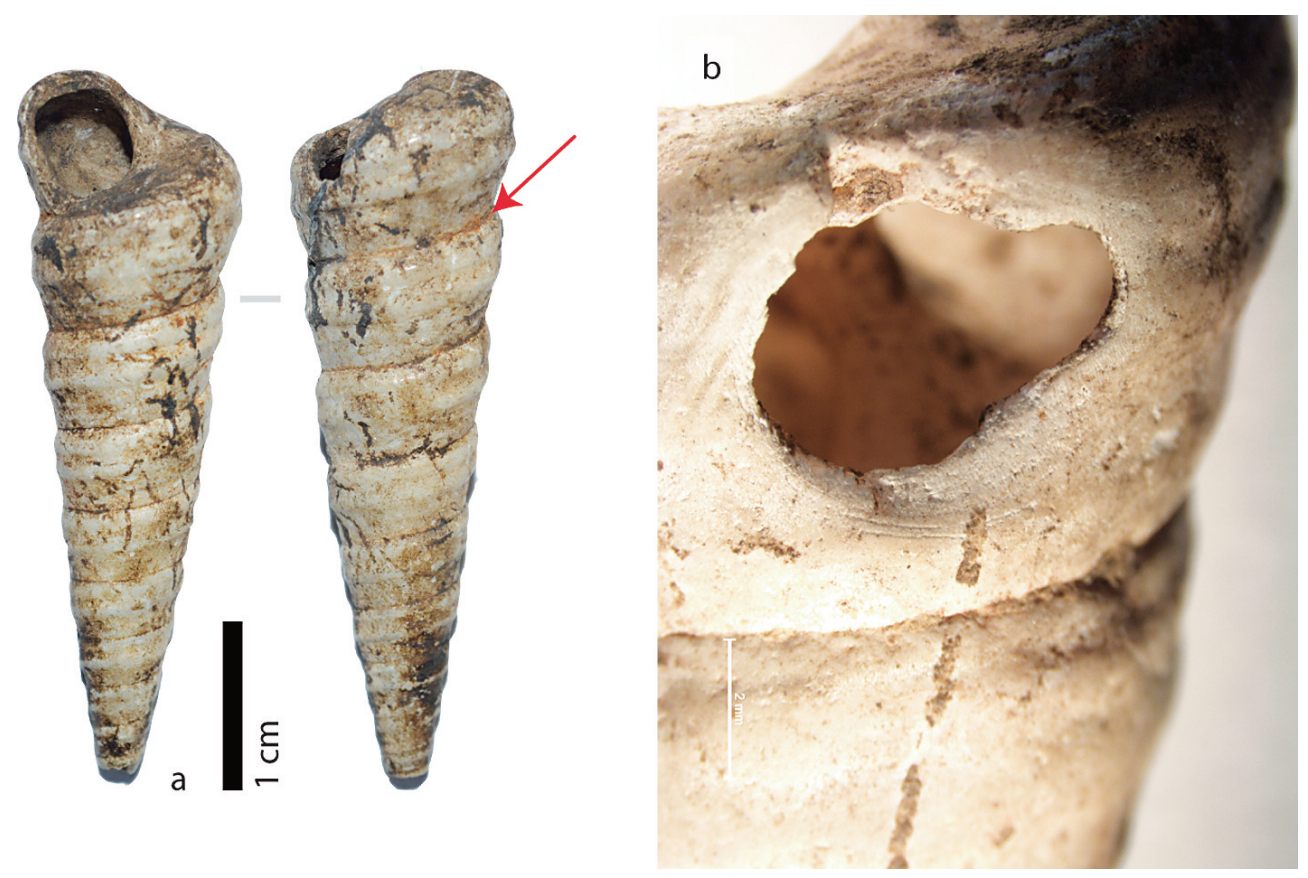

Figure 8 - a) Coquilles de Turritella triplicata présentant une perforation ;

b) perforation ;

flèche) résidus de pigments (Photos E. Campmas).

Figure 8 - a) Shells of Turritella triplicata with a perforation; b) perforation; arrows) pigment residues (Pictures E. Campmas).

des Babors (Arambourg et al. 1934 ; Saxon et al. 1974 ; Merzoug accepté). Nos résultats concernant la malacofaune consommée par les groupes ibéromaurusiens de l'abri Alain viennent donc à la fois confirmer et compléter ces observations. Comme sur la côte méditerranéenne, des données sur la façade atlantique marocaine (grotte des Contrebandiers, Témara, Maroc, fig. 1-3) suggèrent que les groupes ibéromaurusiens ont consommé principalement des Mytilidae et des Patellidae, mais également dans une moindre mesure des Trochidae et des Muricidae (Steele et Alvarez-Fernandez 2011). Notons également la mention d'un reste d'Haliotis à la grotte des Contrebandiers (Steele et Alvarez-Fernandez 2011). De plus, dans sa publication P. Pallary (1934) indique qu'à l'abri Alain les mollusques marins, et notamment ceux consommés, sont principalement présents dans la « couche noire ». II en est de même dans la région des Babors où les coquilles de mollusques sont principalement présentes dans les niveaux supérieurs des stratigraphies (Arambourg et al. 1934 ; Saxon et al. 1974 ; Merzoug accepté) et où, par exemple, l'augmentation des apports halieutiques, avec notamment des Patellidae et Trochidae (principalement Phorcus turbinatus), est observée vers 17-16 ka cal BP à Taza 1 (fig. 1-20, Merzoug accepté). Ces observations vont dans le sens d'une diversification des ressources exploitées, en particulier des mollusques, et notamment des mollusques terrestres, à partir de la phase récente de l'lbéromaurusien (Taylor et al. 2011 ; Lubell 2004 ; Lubell et Barton 2011). À l'abri Alain, P. Pallary (1934 - p. 25) indique également que les mollusques marins sont infiniment moins nombreux que les terrestres, mais une analyse de ces derniers, représentés par de nombreux taxons comestibles (Helix aspersa, Pseudotachea splendida, Sphincterochila candidissima, Otala punctata) et intrusifs (Rumina decollata, Helicidae) (Sidi Maamar 1987) permettrait de mieux appréhender leur participation à l'alimentation des Ibéromaurusiens.

Des coquilles de mollusque marin perforées, collectées dans un but non alimentaire, probablement symbolique, sont mentionnées dans différents sites notamment marocains (Ifri n'Ammar, fig. 1-8, Moser 2003 ; Taforalt, fig. 1-9, Camps 1974 ; Merzoug 2005 d'après Roche 1963), algériens (Mouillah, fig. 1-11, Camps 1974 ; Camps-Fabrer 1994 ; Rassel, fig. 1-17, Brahimi 1970, Camps 1974 ; Camps-Fabrer 1994 ; Afalou-Bou-Rhummel, fig. 1-18, Merzoug 2005 d'après Hachi 2003 ; Tamar-Hat, fig. 1-19, Camps-Fabrer 1994, Merzoug 2005 d'après Hachi 2003) et tunisiens (Oued el-Akarit, fig. 1-22, Camps-Fabrer 1994). Comme à l'abri Alain, elles correspondent principalement à des Turritellidae, Dentaliidae et des Glycymerididae. Cependant, des Columbellidae, Pectinidae, Cardiidae, Spondylidae et Nassariidae sont plus rarement mentionnés. Concernant les Glycymerididae, G. Camps (1974 - p. 99) avait même suggéré que les Ibéromaurusiens utilisaient des "valves de pétoncle déjà usées et parfois naturellement perforées [...] dans de rares cas ils ont tenté d'opérer eux-mêmes la perforation du crochet des coquilles en l'usant par frottement, plus qu'en creusant à l'aide d'un foret ». Ces observations rappellent ce que nous décrivons à l'abri Alain. Si à l'abri Alain, les coquilles ayant été utilisées pour l'ornementation paraissent majoritaires, les fouilles plus récentes d'Afalou Bou Rhummel (1983-1993) et de Taza 1 (1984-1993) ont démontré que les restes de patelles, par exemple, sont les plus nombreuses, en comparaison aux mollusques ne participant pas à l'alimentation (Hachi 2003 ; Merzoug accepté). Notre travail fournit de premières pistes sur les éléments constituant les ornements utilisés à l'Ibéromaurusien, mais seule l'analyse approfondie des 
éléments de parure participera au développement des recherches actuelles sur cette culture visant à cerner son identité, ainsi que les dynamiques de peuplement de l'Afrique du Nord-Ouest.

\section{2 - Comparaisons diachroniques et géographiques dans le bassin méditerranéen}

Les mollusques marins consommés à l'Ibéromaurusien, aussi bien à l'abri Alain que dans la région des Babors, principalement Patellidae, Mytilidae et Trochidae sont similaires à ceux décrits pour le Middle Stone Age d'Afrique du Nord (130-60 ka) sur la façade atlantique marocaine de Témara-Rabat (Steele et Alvarez-Fernandez 2011, 2012 ; Campmas et al. 2016, accepté). Cependant, des différences apparaissent, notamment concernant les Ostreidae, Pectinidae ou Cerastoderma edule, taxons dont le statut à l'abri Alain reste toutefois à préciser. Pour les périodes contemporaines ou sub-contemporaines de l'Ibéromaurusien, ce spectre malacologique est similaire à celui décrit dans d'autres zones géographiques, comme dans les niveaux pré-LGM (Late Glacial maximum), LGM et post-LGM d'Europe et du Levant, par exemple à Riparo Mochi (Italie) (Stiner 1999) et à Ksâr'Akil (Liban) (Curry 2015 ; Bosch et al. 2015). En Libye, dans les niveaux oranien et capsien de Haua Fteah ce sont les Trochidae (Phorcus turbinatus) et les Patellidae (Patella caerulea) qu sont prépondérants (Hunt et al. 2011 ; Prendergast et al. 2016). Alors que dans le site Capsien SHM-1 de la sebkhalagune de Menjel (Tunisie), ce sont les Cardiidae (Cerastoderma glaucum) qui dominent la malacofaune marine consommée, même si les Patellidae, Trochidae, Muricidae, Ostreidae et les Veneridae ont aussi participé à l'alimentation (Mannino et Mazzanti 2013). Dans les sites néolithiques de Tarfaya (Maroc), P. Brebion (1981) suggère que les Hommes ont consommé principalement des Patellidae et des Muricidae, mais également d'autres taxons tels que des Trochidae, des bivalves ou des Volutidae. II note l'absence de transformation de la malacofaune en parure, mais il propose que certains Cymbium cf. tritonis ont en revanche servi de récipient. Au LGM et au post-LGM en Europe, les mollusques de milieux sableux et /ou lagunaire (Ostreidae, Cardiidae) sont aussi décrits en proportions variables, en parallèle à des mollusques marins d'environnement rocheux (Patellidae, Trochidae, Mytilidae) (Aura Tortosa 2002 ; Fa 2008 ; Jorda Pardo 2009 ; Jordá Pardo et al. 2016 ; Colonese et al. 2011 ; Stiner et Munro 2011). A.C. Colonese et al. (2011) suggèrent qu'en Europe, l'intensification de l'exploitation de la malacofaune marine a lieu en parallèle à une intensification des activités de pêche, de la consommation d'oiseaux et de mammifères marins, ainsi que d'une augmentation des distances de transport des ressources marines. En Afrique du Sud, il en est de même. En effet, si l'exploitation des mollusques marins est documentée dès le Middle Stone Age, elle semble s'intensifier au Later Stone Age, avec des densités de coquilles de mollusques marins importantes dans les niveaux archéologiques postérieurs à 10 ka (Jerardino 2016). Le remplissage de l'abri Alain, plus riche en coquilles de mollusques dans sa partie supérieure où des restes de poissons, d'oiseaux aquatiques (anatidés)
(Chibane 2016) sont décrits tout comme les sites des Babors et notamment Taza 1 (Merzoug accepté), pourrait donc s'intégrer à ce modèle d'intensification de l'exploitation des ressources marines, qui serait en lien avec, en Afrique du Nord, la mise en place de dépôts " noirs pulvérulents " datés à environ 16-12 ka cal BP. Toujours en Afrique du Nord, mais au Capsien, cette diversification des ressources exploitées est également caractérisée par la consommation d'un grand nombre de mollusques terrestres (Lubell 2004 ; Colonese et al. 2011), ce qui semblait déjà s'amorcer dans la phase récente de l'Ibéromaurusien (Taylor et al. 2011). Toutefois, il est à noter que si les amas pulvérulents riches en mollusques terrestres et/ou marins sont attribués au Capsien au nordest de l'Afrique du Nord-Est (Lubell 2004), ils semblent également être une caractéristique de la fin de l'Ibéromaurusien à l'ouest de l'Algérie ou à l'est du Maroc (e.g. Pallary 1934 ; Camps 1974 ; Moser 2003 ; Barton et al. 2013). C'est également le cas pour les occupations néolithiques des zones côtières de la façade atlantique marocaine, riches en coquilles de moules (Nespoulet et al. 2008).

À l'abri Alain, aucune coquille de Nassarius n'a été observée, alors que ce coquillage a été largement utilisé à l'Atérien (Middle Stone Age d'Afrique du Nord) comme ornement (Vanhaeren et al. 2006 ; Bouzouggar et al. 2007 ; d'Errico et al. 2009 ; Nami et Moser 2010 ; Dibble et al. 2012 ; Eiwanger et al. 2012 ; El Hajraoui, Oudouche, Nespoulet 2012 ; Bar-Yosef Mayer 2015). Toutefois, quelques mentions de Nassariidae sont faites à l'lbéromaurusien, notamment à l'oued el-Akarit (Tunisie) (Camps 1974). Cependant, dans cette localité, en parallèle à la description de stations « à lamelles » il est fait mention également d'un gisement Middle Stone Age (Zoughlami et al. 1985), ce qui soulève des questions sur l'utilisation des Nassarius aussi bien par les groupes Middle Stone Age qu'ibéromaurusiens, la pollution du site ibéromaurusien par des apports atériens ou le ramassage de Nassarius atériens par les Ibéromaurusiens. Un coquillage de la famille des Nassariidae est également mentionné à la grotte Rassel (fig. 1-17), mais il s'agirait d'un Nassarius granum d'après Brahimi (1970). Des Nassariidae sont décrits à Tamar Hat avec une faible fréquence, mais dans tous les niveaux, toutefois cette famille est uniquement représentée par le genre Tritia (Tritia reticulata, Tritia incrassata et Tritia cf. mutabilis) (Saxon et al. 1974). À l'Atérien il semble que ce soient les Nassarius gibbosulus et/ou Nassarius circumcinctus qui soient prépondérants. Des Nassariidae (Nassarius nitidus, Nassarius gibbosulus, Nassarius mutabilis) sont toutefois décrits comme ornement dans le site Capsien de SHM-1 (Mannino et Mazzanti 2013) et un exemplaire complètement poli jusqu'à la columelle a été identifié dans le site Capsien d'El Mekta (Gobert 1951 ; Rahmani 2003). Les Nassariidae semblent donc avoir été utilisés comme ornement en Afrique du Nord à différentes périodes. La principale différence entre les ornements atériens et ibéromaurusiens en malacofaune marine semble donc résider dans la diversité des taxons utilisés plutôt que dans leur nature. Cette diversité s'avère beaucoup plus importante à l'lbéromaurusien ainsi qu'au 
Capsien ou au Néolithique ancien d'Afrique du Nord (Camps-Fabrer 1994 ; Mannino et Mazzanti 2013 ; Kherbouche et al. 2014). Notons, que dans un niveau néolithique ancien (avec de la céramique cardiale) d'Ifri Ouzabour (Rif Oriental, Maroc), une coquille de Glycymerididae a également été utilisée comme ornement. Cette coquille perforée est accompagnée de deux autres coquilles de Glycymerididae non perforées (Nekkal et al. 2015). D'après Nekkal et al. (2015) la perforation au niveau du crochet a été produite par percussion, ce qui rappelle les observations que nous avons réalisées à l'abri Alain. Cette diversité s'observe également au Paléolithique supérieur et l'Epipaléolithique du Levant et d'Europe [e.g. Ucagizli cave (Turquie) (Stiner et al. 2002 ; Stiner 2003), Riparo Mochi (Italie) (Stiner 1999), Ksâr'Akil (Liban) (Bosch et al. 2015)] ou encore pour le Mésolithique et le Néolithique d'Europe (Rigaud 2011). Notons de très fortes similitudes de l'aspect taphonomique de la malacofaune d'occupation néolithique d'Espagne (Caserna de Sant Pau del Camp) avec l'aspect des Glycymerididae de l'abri Alain, qui sont complets ou non et érodés après fragmentation dans les deux cas. L. Lloveras et al. (2014) indiquent que leur présence à la Caserna de Sant Pau del Camp ne semble pas s'accorder avec les données géomorphologiques et la distribution spatiale. Celles-ci suggèrent qu'elles ne s'apparentent pas à un dépôt de front de tempête. À l'abri Alain, les dépôts, en particulier la couche noire pulvérulente, ne semblent pas non plus s'apparenter à un dépôt de tempête. L. Lloveras et al. (2014) estiment que leur concentration plus élevée autour des sépultures pourrait attester de leur utilisation comme ornement. Néanmoins, ces coquilles ne sont pas directement présentes dans le dépôt sépulcral, et aucune concentration spatiale n'a pu être liée à des zones d'atelier. Nous proposons également à l'abri Alain leur utilisation pour l'ornementation. En revanche, si F. Doumergue (1935) mentionne la présence de deux restes humains (phalanges) aucune sépulture n'est signalée. Leur utilisation comme offrande en lien avec des structures sépulcrales semble donc pouvoir être éliminée à l'abri Alain. L. Lloveras et al. (2014) suggèrent qu'en Espagne ces coquilles auraient pu servir à la production de bracelets, alors que la présence de perforations à l'abri Alain pourrait plutôt évoquer leur utilisation en suspension. Cette différence de fonction pourrait être liée à des variations chronologiques, géographiques, fonctionnelles des sites ou même aux méthodes de fouille, avec à l'abri Alain, l'absence potentielle de certains éléments. De plus, il est également à noter qu'à Aviones (Espagne), J. Zilhão et al. (2010) suggèrent que les Néandertaliens ont utilisé différents supports de parure, dont Acanthocardia tuberculata et Glycymeris nummaria, comme à l'abri Alain. Enfin, un autre élément intéressant concerne les scaphopodes, qui occupent une place importante à l'abri Alain et qui se rencontrent également au Natoufien (Levant, 14.5$11.5 \mathrm{ka}$ cal BP), au cours duquel les mollusques marins et les poissons ont également été consommés (Bar-Yosef Mayer 2008 ; Bar-Yosef Mayer et Zohar 2010). Ces observations soulèvent donc des questions à la fois sur des phénomènes de convergences/récurrences comportementales par des lignées humaines différentes à des périodes distinctes dans des environnements similaires, sur la fiabilité des séquences stratigraphiques, ou sur des phénomènes de diffusion (de matériaux, idées et/ou populations) autour du bassin méditerranéen entre 16 et 12 ka cal BP.

\section{Conclusion}

Si la collection malacologique de l'abri Alain provient de fouille du début du $20^{\text {e }}$ siècle et a subi une histoire complexe traduisant peut-être différentes périodes d'occupation, elle nous permet cependant de fournir des résultats inédits sur les comportements de subsistance et symboliques des Ibéromaurusiens. Les groupes ibéromaurusiens de l'abri Alain ont collecté les mollusques de biotopes rocheux (Patellidae, Mytilidae, Trochidae principalement) dans la zone intertidale, probablement lors des marées basses. Les Muricidae, Ostreidae, Pectinidae, Cerastoderma edule pourraient également avoir été consommés, mais leur statut reste à préciser. Les Ibéromaurusiens ont aussi utilisé une grande diversité de coquilles de mollusques marins, ramassées en position secondaire, pour réaliser des parures (Dentaliidae, Glycymerididae, Cardiidae, Turritellidae). Pour l'Ibéromaurusien, cette collection provenant de la région oranaise fait apparaître des similitudes importantes également avec la région des Babors (Merzoug accepté). Si l'exploitation des mollusques, aussi bien à des fins alimentaires que symboliques, est attestée dès le Middle Stone Age / Paléolithique Moyen, elle semble s'intensifier au Later Stone Age / Paléolithique Supérieur / Epipaléolithique, autant en Afrique qu'en Europe. Le dépôt noir pulvérulent riche en coquilles de mollusque de l'abri Alain, daté vers 15-12 ka cal. BP, semble donc s'intégrer à ce modèle. Néanmoins, si ce réexamen fournit des pistes sur la caractérisation de la malacofaune marine consommée par les Ibéromaurusiens, des recherches menées sur des séries provenant de fouilles exhaustives permettant d'appréhender la diversité spécifique, la sélection des individus, les modalités de collecte, de traitement et de préparation, ainsi que les dégradations post- dépositionnelles des coquilles doivent être entreprises. Concernant la malacofaune utilisée pour l'ornementation, nous avons repéré que certains éléments présentent des traces de façonnage et d'utilisation associées à la présence de résidus de pigments. Néanmoins, pour une meilleure compréhension des marqueurs identitaires, des systèmes symboliques des groupes ibéromaurusiens, leur analyse reste à affiner (supports sélectionnés, modalité de transformation et usures d'utilisation).

\section{Remerciements}

Nous exprimons toute notre gratitude à $\mathrm{H}$. De Lumley, St. et R. Orliac pour nous avoir permis d'accéder aux collections de l'Institut de Paléontologie Humaine. Nos vifs remerciements vont également à $\mathrm{C}$. Daujeard et L. Crépin, pour leurs relectures, leurs commentaires, ainsi que pour l'accès à la loupe binoculaire à l'IPH de C. Daujeard. Nous remercions E. Stoetzel pour nous avoir transmis les 
données inédites concernant l'analyse de la microfaune, ainsi que pour sa relecture et ses commentaires. Nous remercions $\mathrm{S}$. Chibane pour les discussions que nous avons eues autour de l'Ibéromaurusien et de l'exploitation des vertébrés terrestres de l'abri Alain. Nous remercions particulièrement $P$. Granchon (INRAP) pour nous avoir transmis son mémoire de Maîtrise, ainsi que L. Sari pour son aide. Toute notre gratitude également à $\mathrm{M}$. Cammas pour l'infographie de la figure 1. Nous remercions les deux relecteurs, M. Nami et le second relecteur anonyme pour leurs relectures et conseils avisés.

\section{Références bibliographiques}

ABBAR H. 2014 - La faune ibéromaurusienne de la couche jaune de l'Abri Alain (Oran, Algérie): Etude Paléontologique et Paléoécologique. Paris : Muséum National d'Histoire Naturelle. Mémoire de Master 1.

ALDEIAS V., GUR-ARIEH S., MARIA R., MONTEIRO P. et CURA P. 2016 - Shell we cook it? An experimental approach to the microarchaeological record of shellfish roasting. Archaeological Anthropological Science, 1-19.

AOUDIA-CHOUARKI L. 2013 - Pratiques funéraires complexes: réévaluation archéo-anthropologique des contextes ibéromaurusiens et capsiens (Paléolithique supérieur et Epipaléolithique, Afrique du Nord-Ouest). Bordeaux : Université Bordeaux 1. Thèse de doctorat.

ARAMBOURG C. 1927 - Les Poissons Fossiles d'Oran. Matériaux pour la Carte Géologique de l'Algérie, 6, 298 p.

ARAMBOURG C., BOULE M., VALLOIS H., VERNEAU R. 1934 - Les grottes paléolithiques des Beni Ségoual (Algérie). Archives de l'Institut de Paléontologie Humaine, 13, $242 \mathrm{p}$.

AURA TORTOSA J.E. 2002 - The far south: the Pleistocene-Holocene transition in Nerja Cave (Andalucía, Spain). Quaternary International, 93-94, p. 19-30.

BALOUT L. 1950 - Préhistoire de l'Afrique du Nord essai de chronologie. Paris : Arts et métiers graphiques.

BARTON R.N.E., BOUZOUGGAR A., COLLCUTT S.N., CARRIÓN MARCO Y., CLARK-BALZAN L., DEBENHAM N.C., MORALES J. 2016 - Reconsidering the MSA to LSA transition at Taforalt Cave (Morocco) in the light of new multi-proxy dating evidence. Quaternary International, 413, p. 36-49.

BARTON N., BOUZOUGGAR A., COLLCUTT S.N., HIGHAM T.F.G., HUMPHREY L.T., PARFITT S., RHODES E., SCHWENNINGER J.-L., STRINGER C., TURNER E., WARD S. 2007 - Abrupt Climatic Change and Chronology of the Upper Palaeolithic in Northern and Eastern Morocco. In : P. Mellars, K. Boyle., O. Bar-Yosef, C. Stringer (Ed.), Rethinking the Human Revolution New Behavioural and Biological Perspectives on the Origin and Dispersal of Modern Humans. Oxford : Oxbow Books, p. 177-190.
BARTON R.N.E., BOUZOUGGAR A., HOGUE J.T., LEE S., COLLCUTT S.N. et DITCHFIELD P. 2013 - Origins of the Iberomaurusian in NW Africa: New AMS radiocarbon dating of the Middle and Later Stone Age deposits at Taforalt Cave, Morocco. Journal of Human Evolution, 65, p. 266-281.

BARTON N., BOUZOUGGAR A., HUMPHREY L., BERRIDGE P., COLLCUTT S., GALE R., PARFITT S., PARKER A., RHODES E. et SCHWENNINGER J.-L. 2008 - Human Burial Evidence from Hattab II Cave and the Question of Continuity in Late Pleistocene-Holocene Mortuary Practices in Northwest Africa. Cambridge Archaeological Journal, 18, p. 195-214.

BAR-YOSEF MAYER D.E. 2008 - Dentalium Shells Used by Hunter-Gatherers and Pastoralists in the Levant. Archaeofauna, 17, p. 103-110.

BAR-YOSEF MAYER D.E. 2015 - Nassarius shells: Preferred beads of the Palaeolithic. Quaternary International, 390, p. 79-84.

BAR-YOSEF MAYER D.E., ZOHAR I. 2010 - The role of aquatic resources in the Natufian Culture. Eurasian Prehistory, 7, p. 29-43.

BOSCH M.D., MANNINO M.A., PRENDERGAST A.L., O'CONNELL T.C., DEMARCHI B., TAYLOR S.M., NIVEN L., PLICHT J. VAN DER et HUBLIN J.-J. 2015 - New chronology for Ksâr 'Akil (Lebanon) supports Levantine route of modern human dispersal into Europe. Proceeding of National Acadademic Sciences of United States of America, 112, p. 7683-7688.

BOUZOUGGAR A., BARTON R.N.E., BLOCKLEY S., BRONK-RAMSEY C., COLLCUTT S.N., GALE R., HIGHAM T.F.G., HUMPHREY L.T., PARFITT S., TURNER, E. et WARD S. 2008 - Reevaluating the Age of the Iberomaurusian in Morocco. African Archaeological Review, 25, p. 3-19.

BOUZOUGGAR A., BARTON N., VANHAEREN M., D'ERRICO F., COLLCUTT S., HIGHAM T., HODGE E., PARFITT S., RHODES E., SCHWENNINGER J.-L., STRINGER C., TURNER E., WARD S., MOUTMIR A. et STAMBOULI A. 2007 - 82,000-year-old shell beads from North Africa and implications for the origins of modern human behavior. Proceeding of National Academic Sciences of United States of America, 104, p. 9964-9969.

BRAHIMI C. 1970 - L'Ibéromaurusien du littoral de la région d'Alger, Alger : Mémoire du CRAPE, 23, 154 p.

BREBION P. 1981 - Les gastéropodes du Néolithique de Tarfaya (Maroc). In : Préhistoire Africaine-Mélanges offerts au Doyen Lionel Balout, p. 253-256.

BUCQUOY D.E., DAUTZENBERG P., DOLLFUS G. 18821886 - Mollusques marins du Roussillon, Tome ler, Gastéropodes, Paris : J.B. Baillières \& Fils. 
BUCQUOY D.E., DAUTZENBERG P., DOLLFUS G. 18871898 - Mollusques marins du Roussillon, Tome II, Pélécypodes, Paris : J.B. Baillières \& Fils.

CABRAL J.P. et MARTINS J.M.S. 2016 - Archaeological Glycymeris glycymeris shells perforated at the umbo: Natural or man-made holes? Journal of Archaeological Science: Reports, p. 474-482.

CAMPMAS E., AMANI F., MORALA A., DEBÉNATH A., EL HAJRAOUI M.A. et NESPOULET R. 2016 - Initial insights into Aterian hunter-gatherer settlements on coastal landscapes: The example of Unit 8 of El Mnasra Cave (Témara, Morocco). Quaternary International, 413, p. 5-20.

CAMPMAS E., CHAKROUN A., CHAHID D., LEBON M., EL HAJRAOUI M.A., NESPOULET R. accepté Subsistance en zone côtière durant le Middle Stone Age en Afrique du Nord: Exemple des données préliminaires sur l'Unité 8 de la grotte d'El Mnasra (Témara, Maroc), In : Actes Du $141^{\circ}$ Congrès Du CTHS, Rouen 2016.

CAMPS G. 1974 - Les Civilisations préhistoriques de l'Afrique du Nord et du Sahara. Paris : Doin.

CAMPS-FABRER H. 1994 - Coquillages. In : G. Camps (Ed.), Encyclopédie berbère. Louvain : Peeters, p. 2088-2092.

CHAKROUN A. 2006 - Étude sédimentologique et paléontologique des affleurements du Quaternaire le long de la côte Nord Orientale de la Tunisie. Tunis : Université de Tunis - Perpignan : Université de Perpignan. Thèse de doctorat.

CHAKROUN A., ZAGHBIB-TURKI D. sous presse - Facies and fauna proxies used to reconstruct the MIS 5 and MIS 7 coastal environments in eastern Tunisia. Geological Quarterly.

CHAKROUN A., ZAGHBIB-TURKI D., MISKOVSKY J. C., DAVAUD E. 2009 - Two transgressive cycles in coastal deposits of the Cap Bon peninsula, Tunisia. Quaternaire, 20 , p. $215-226$.

CHAKROUN A., ZAGHBIB-TURKI D., MONCEF TURKI M. 2016 - The Upper Pleistocene deposits in Rafraf (Northeastern Tunisia): new data on the Persististrombus latus level. Arabian Journal of Geosciences, 9, p. 1-13.

CHIBANE S. 2016 - Étude taphonomique et archéozoologique de la faune ibéromaurusienne de l'abri Alain (Oran, Algérie). Toulouse : Université Toulouse Jean Jaurès. Mémoire de Master 2.

COLONESE A.C., MANNINO M.A., BAR-YOSEF MAYER D.E., FA D.A., FINLAYSON J.C., LUBELL D., STINER M.C. 2011 - Marine mollusc exploitation in Mediterranean prehistory: An overview. Quaternary International, 239, p. 86-103.

CURRY A. 2015 - Ancient humans brought tools to Europe. Nature-News.
DE GROOTE I. et HUMPHREY L.T. 2015 - Characterizing evulsion in the Later Stone Age Maghreb: Age, sex and effects on mastication. Quaternary International, 413, p. 50-61.

D'ERRICO F., VANHAEREN M., BARTON N., BOUZOUGGAR A., MIENIS H., RICHTER D., HUBLIN J.-J., MCPHERRON S., LOZOUET P. 2009 - Additional evidence on the use of personal ornaments in the Middle Paleolithic of North Africa. Proceeding of National Academic Sciences of United States of America, 106, p. 16051-16056.

DIBBLE H., ALDEIAS V., ALVAREZ-FERNANDEZ E., BLACKWELL B., HALLETT-DESGUEZ E., JACOBS Z., GOLDBERG P., LIN S., MORALA A., MEYER M., OLSZEWSKI D., REED K., REZEK Z., RICHTER D., ROBERTS R., SANDGATHE D., SCHURMANS U., SKINNER A., STEELE T., EL-HAJRAOUI M. 2012 - New excavations at the site of Contrebandiers Cave, Morocco. PaleoAnthropology, 145-201.

DOUMERGUE F. 1935 - Grotte démantelée des carrières d'Eckmühl. Bulletin de la Société de géographie et d'archéologie de la province d'Oran.

DUPONT C. 2006 - La malacofaune de sites mésolithiques et néolithiques de la façade atlantique de la France: Contribution à l'économie et à l'identité culturelle des groupes concernés. BAR International Series 1571.

DUPONT C. 2014 - Do not mix up apples and oranges! A concept applied to shells from the Mesolithic. In : J.-J. Cantillo, D. Bernal, J. Ramos (Ed.), Moluscos y purpura en contextos arqueologicos atlantico-mediterraneos: Nuevos datos $y$ reflexiones en clave de proceso historico. Actas de la III reunion Cientifica de la peninsula Iberica, Cádiz (décembre 2012), Cádiz, Servicio de Publicaciones de la Universidad de Cádiz, p. 41-50.

EIWANGER J., MIKDAD A., MOSER J. et NAMI M. 2012 Découverte de coquilles perforées de type Nassarius au site Ifri n'Ammar (Rif Oriental, Maroc). Bulletin d'Archéologie Marocaine, XXII, p. 9-15.

EL HAJRAOUI M.A., OUDOUCHE H. et NESPOULET R. 2012 - Etude des coquilles perforées découvertes à Témara. In : M.A El Hajraoui, R. Nespoulet, A. Debénath, H. L. Dibble (Ed.), La Préhistoire de La Région de RabatTémara. Rabat : Villes et sites Archéologiques du Maroc, Vol. III, Rabat : Publication du Ministère de la Culture et de l'Institut National des Sciences de l'Archéologie et du Patrimoine, p. 191-199.

ESU D. et GIROTTI O. 2010 - The late Oligocene molluscan fauna from Otranto (Apulia, southern Italy): an example of alternating freshwater, lagoonal and emerged environments, 53, p. 137-174.

FA D.A. 2008 - Effects of tidal amplitude on intertidal resource availability and dispersal pressure in prehistoric human coastal populations: the Mediterranean-Atlantic transition. Quaternary Science Review, 27, p. 2194-2209. 
FEKI M. 1975 - Paléoécologie du Pliocène marin au Nord de la Tunisie. Annales des Mines et de la Géologie, 27, $194 p$

FRENEIX S., SAINT MARTIN J.-P. et MOISSETTE P. 1987 - Bivalves Ptériomorphes du Messinien d'Oranie (Algérie occidentale), Bulletin du Muséum National d'Histoire Naturelle, Paris, 9, p. 3-61.

GENTIL L. 1903 - Étude géologique du bassin de la Tafna. Bulletin du Service des Cartes Géologiques de l'Algérie, 4, $425 \mathrm{p}$.

GOBERT E.G. 1951 - El Mekta, station princeps du Capsien. Karthago, III, p. 1-79.

GRANCHON P. 1987 - L'industrie ibéromaurusienne de l'abri Alain, Oran-Algérie. Paris : Université de Paris I. Mémoire de maîtrise.

HACHI S. 2003 - Les cultures de l'Homme de MechtaAfalou. Le gisement d'Afalou-Bou-Rhummel (massif des Babors, Algérie), les niveaux supérieurs 13 000-11 000 BP. Mémoire du Centre National de Recherches Préhistoriques Anthropologiques et Historiques, 2, $256 \mathrm{p}$.

HADJOUIS D. 2002 - Les hommes du Paléolithique supérieur d'Afalou Bou Rhummel (Bedjaia, Algérie). Interprétation nouvelle des cinétiques cranio-faciales et des effets de l'avulsion dentaire. Malformations crâniennes, troubles de la croissance, anomalies et maladies alvéolodentaires. L'Anthropologie, 106, p. 337-375.

HELLAL H. 2005. L'Ibéromaurusien de l'abri Alain (Algérie occidentale). Approche Technotypologique des industries lithiques. Paris : Université de Paris I. Mémoire de DEA.

HOGUE J.T., BARTON R.N.E. 2016 - New radiocarbon dates for the earliest Later Stone Age microlithic technology in Northwest Africa. Quaternary International, 413, p. 62-75.

HÖRNES M. 1852 -1856 - Die fossilen Mollusken des Tertiär-Beckens von Wien. I. Band. Univalven. Abh. K.K Geol. Reichsanst, 1-10, 736 p.

HUMPHREY L.T., BELLO S.M., TURNER E., BOUZOUGGAR A. et BARTON N. 2012 - Iberomaurusian funerary behaviour: Evidence from Grotte des Pigeons, Taforalt, Morocco. Journal of Human Evolution, 62 p. 261-273.

HUMPHREY L.T., BOCAEGE E. 2008 - Tooth Evulsion in Maghreb: Chronological and Geographical Patterns. African Archaeological Review, 25, p. 109-123.

HUMPHREY L.T., GROOTE I.D., MORALES J., BARTON N., COLLCUTT S., RAMSEY C.B., BOUZOUGGAR A. 2014 - Earliest evidence for caries and exploitation of starchy plant foods in Pleistocene huntergatherers from Morocco. Proceeding of National Academic Sciences of United States of America, 111, p. 954-959.
HUNT C.O., REYNOLDS T.G., EL-RISHI H.A., BUZAIAN A., HILL E., BARKER G.W. 2011 - Resource pressure and environmental change on the North African littoral: Epipalaeolithic to Roman gastropods from Cyrenaica, Libya. Quaternary International, 244, p. 15-26.

HUVET A. 2000 - Ressources génétiques et phylogéographie des huîtres creuses Crassostrea gigas et Crassostrea angulata: variabilité, différenciation et adaptation des populations naturelles et introduites. Tours : Université François Rabelais. Thèse de doctorat.

JERARDINO A. 2016 - On the origins and significance of Pleistocene coastal resource use in southern Africa with particular reference to shellfish gathering. Journal of Anthropological Archaeology, 41, p. 213-230.

JORDA PARDO J. 2009 - La malacofauna de la Cueva de Nerja (III): Evolución medioambiental y técnicas de marisqueo. Zephyrvs, 37.

JORDA PARDO J.F., AURA TORTOSA J.E., AVEZUELA ARISTU B., ÁLVAREZ-FERNANDEZ E., GARCIA-PEREZ A. et MAESTRO A. 2016 - Breaking the waves: Human use of marine bivalves in a microtidal range coast during the Upper Pleistocene and the Early Holocene, Vestíbulo chamber, Nerja Cave (Málaga, southern Spain). Quaternary International, 407, p. 59-79.

KHERBOUCHE F., HACHI S., ABDESSADOK S., SEHIL N., MERZOUG S., SARI L., BENCHERNINE R., CHELLI R., FONTUGNE M., BARBAZA M., ROUBET C. 2014 Preliminary results from excavations at Gueldaman Cave GLD1 (Akbou, Algeria). Quaternary International, 320, 109-124.

LINDNER G. 2015 - Coquillages marins: Plus de 1000 espèces des mers du monde, Paris : Delachaux et Niestlé.

LINSTÄDTER J. 2008 - The Epipalaeolithic-NeolithicTransition in the Mediterranean region of Northwest Africa. Quartär, 55, p. 33-54.

LINSTÄDTER J., EIWANGER J., MIKDAD A., WENIGER G.-C. 2012 - Human occupation of Northwest Africa: A review of Middle Palaeolithic to Epipalaeolithic sites in Morocco. Quaternary International, 274, 158-174.

LLOVERAS L., VICENTE O., MOLIST M., NADAL J., RIERA S., JULIÀ R., ESTRADA A. 2014 - Interpretación tafonómica de la malacofauna marina en el yacimiento neolítico de la Caserna de Sant Pau del Camp (Barcelona). Archaeofauna, 23, 169-179.

LOCARD A. 1892 - Les coquilles marines des côtes de France: Description des familles, genres et espèces, Paris : Librairie J.B. Baillières et Fils.

LOPPENS K. 1926 - La perforation des coquilles de Mollusques par des gastéropodes et des éponges. Annale Société Royale Zoologique de Belgique., LVII, p. 14-18. 
LUBELL D. 2004 - Prehistoric edible land snails in the circum-Mediterranean: the archaeological evidence. In : J. Ph. Brugal, J. Desse (Ed.), Petits Animaux et Sociétés Humaines. Du Complément Alimentaire Aux Ressources Utilitaires. XXIV Rencontres Internationales D'archéologie et D'histoire d'Antibes. Antibes (octobre 2003), Antibes, APDCA, p. 77-98.

LUBELL D. et BARTON N. 2011 - Gastropods and humans in the late Palaeolithic and Mesolithic of the western Mediterranean basin. Quaternary International, 244, p. 1-4.

MANNINO M.A. et MAZZANTI C. 2013 - Studi ed osservazioni preliminari sulla malacofauna dal sito capsiano costiero SHM-1 (Tunisia centrale). In : S. Mulazzani (Ed.), Le Capsien de Hergla (Tunisie): Culture, Environnement et Économie. Altkönigblick : Africa Magna Verlag, p. 339-361.

MARIOTTI V., BELCASTRO M.G., CONDEMI S. 2016 From corpse to bones: funerary rituals of the Taforalt Iberomaurusian population. BMSAP, 28, 60-65.

MARIOTTI V., BONFIGLIOLI B., FACCHINI F., CONDEMI S. et BELCASTRO M.G. 2009 - Funerary practices of the Iberomaurusian population of Taforalt (Tafoughalt; Morocco, 11-12,000BP): new hypotheses based on a grave by grave skeletal inventory and evidence of deliberate human modification of the remains. Journal of Human Evolution, 56, p. 340-354.

MARIOTTI V., CONDEMI S., BELCASTRO M.G. 2014 Iberomaurusian funerary customs: new evidence from unpublished records of the 1950s excavations of the Taforalt necropolis (Morocco). Journal of Archaeological Science, 49, p. 488-499.

MERZOUG S. 2005 - Comportement de subsistance des Ibéromaurusiens d'après l'analyse archéozoologique des mammifères de Tamar Hat, Taza 1 et Columata (Algérie). Paris : Muséum National d'histoire Naturelle. Thèse de doctorat.

MERZOUG S. 2008 - Étude archéozoologique des mammifères ibéromaurusiens de la collection Cadenat de Columnata (Tiaret, Algérie). In : H. Aouraghe, H. Haddoumi, K. El Hammouti, A. Oujda (Ed.), Le Quaternaire Marocain Dans Son Contexte Méditerranéen. Actes des quatrièmes rencontres des Quaternaristes marocaines (RQM4), Oujda (novembre 2007), Oujda, Publication de la Faculté des Sciences, Université Mohammed 1 1', p. 255-264.

MERZOUG S. accepté - Les comportements de subsistance en Afrique du Nord-Ouest durant la transition Pléistocène supérieur / Holocène : entre homogénéité et variations stratégiques. L'Anthropologie.

MERZOUG S., SARI L. 2008 - Re-examination of the Zone I Material from Tamar Hat (Algeria): Zooarchaeological and Technofunctional Analyses. African Archaeological Review, 25, p. 57-73.
MEZIANE K., KERFOUF A. 2013 - Biodiversité et distribution spatiale des mollusques de l'estran de la côte Ouest algérienne (cas des substrats durs). In : Actes Du 3ème Colloque International Sur La Biodiversité et Ecosystèmes Littoraux. 26-28 Novembre 2013, Oran, p. $97-104$

MOSER J. 2003 - La Grotte d'Ifri n'Ammar: Tome 1 L'Ibéromaurusien, Köln : Liden Soft.

NAMI M. et MOSER J. 2010 - La grotte d'Ifri n'Ammar: Le Paléolithique moyen, Verlag : Forschungenzur Archäologie Außereuropäischer Kulturen.

NEKKAL F., LINSTÄDTER J., AOURAGHE $\mathrm{H}$. et BOUGARIANE B. 2015 - Ifri Ouzabour : l'extrême extension de la culture cardiale sur le littoral méditerranéen sud (Rif Oriental, Maroc). Bulletin de l'Institut Scientifique, Rabat, Section Science de la Terre, 37, p. 55-76.

NESPOULET R., DEBENATH A., EL HAJRAOUI M.A., MICHEL P., CAMPMAS E., OUJAA A., BEN-NCER A., LACOMBE J.-P., STOETZEL E. et BOUDAD L. 2008 - Le contexte archéologique des restes humains atériens de la région de Rabat-Témara (Maroc): Apport des fouilles des grottes d'El Mnasra et d'El Harhoura 2. In : H. Aouraghe, H. Haddoumi, K. El Hammouti, A. Oujda (Ed.), Le Quaternaire Marocain Dans Son Contexte Méditerranéen. Actes des quatrièmes rencontres des Quaternaristes marocaines (RQM4), Oujda (novembre 2007), Oujda, Publication de la Faculté des Sciences, Université Mohammed 1 1 ${ }^{\mathrm{er}}$, p. 356-375.

NOUET J., CHEVALLARD C., FARRE B., NEHRKE G., CAMPMAS E., STOETZEL E., EL HAJRAOUI M.A. et NESPOULET R. 2015 - Limpet shells of the Aterian level 8 of El Harhoura 2 Cave (Témara, Morocco): Preservation state of crossed-foliated layers. PlosOne, 10, e0137162.

PALLARY P. 1934 - L'abri Alain, près d'Oran, Paris : Masson et Cie.

PASCAL M., CLERGEAU P., LORVELEC O. 2010 Invasions biologiques et biologie de la conservation :essai de synthèse. Courrier de l'environnement de I'INRA, 40, p. 23-31.

PERRIER R. 1954 - La faune de France, IXphioxus Tuniciers). Paris : Delagrave

POITRAT-TARGOWLA M.J. 1977 - Pathologie dentomaxillaire comparée des Hommes d'Afalou et de Taforalt (Ibéromaurusiens du Maghreb). Bulletin des Mémoires de la Société d'Anthropologie de Paris, 4, p. 389-394.

POTIEZ V.L.V. et MICHAUD A.L.G. 1838 - Galerie des mollusques ou catalogue méthodique et coquilles $d u$ Muséum de Douai. Paris : J.B. Baillières \& Fils.

PRENDERGAST A.L., STEVENS R.E., O'CONNELL T.C., FADLALAK A., TOUATI M., AL-MZEINE A., SCHÖNE B.R., HUNT C.O., BARKER G. 2016 - Changing 
patterns of eastern Mediterranean shellfish exploitation in the Late Glacial and Early Holocene: Oxygen isotope evidence from gastropod in Epipaleolithic to Neolithic human occupation layers at the Haua Fteah cave, Libya. Quaternary International, 407, p. 80-93.

RAHMANI N. 2003 - Le Capsien typique et le Capsien supérieur: évolution ou contemporanéité. Bordeaux : Université Bordeaux 1. Thèse de doctorat.

RAMPAL J. 1965 - Utilisation des dents radulaires pour la systématique des Patelles Méditerranéennes. Revue des Travaux de l'Institut de pêche maritime, 29, p. 205-210.

RIGAUD S. 2011 - La parure: traceur de la géographie culturelle et des dynamiques de peuplement au passage Mésolithique-Néolithique en Europe. Bordeaux : Université Bordeaux 1. Thèse de doctorat.

ROCHE J. 1963 - L'Épipaléolithique marocain, Lisbonne : Librairie Bertrand.

ROGER J. 1942 - Note préliminaire sur le Sahélien. Les Invertébrés de la macro faune sahélienne d'Oran. Bulletin du Muséum National d'Histoire Naturelle, Paris, 14 , p. $465-470$.

RUSSELL N., BONSALL C., SUTHERLAND D. 1995 - The exploitation of marine molluscs in the Mesolithic of western Scotland: evidence from Ulva Cave, Inner Hebrides. In: A. Fischer (Ed.), Man and Sea in The Mesolithic. Oxford: Oxbow Book, p. 273-288.

SACCO F. 1890-1904 - I Molluschi dei terreni terziari del Piemonte et della Liguria. Torin : Stamperia Beal.

SARI L. 2008 - Production lamellaire en contexte ibéromaurusien: variabilité et implication technoéconomique. Cas du niveau inférieur de Rassel (littoral ouest algérois). In : H. Aouraghe, H. Haddoumi, K. El Hammouti, A. Oujda (Ed.), Le Quaternaire Marocain Dans Son Contexte Méditerranéen. Actes des quatrièmes rencontres des Quaternaristes marocaines (RQM4), Oujda (novembre 2007), Oujda, Publication de la Faculté des Sciences, Université Mohammed 1', p. 376-388.

SARI L. 2012 - L'Ibéromaurusien, culture du Paléolithique supérieur tardif : approche technologique des productions lithiques taillées de Tamar Hat, Rassel et Columnata (Algérie). Paris : Paris 10. Thèse de doctorat.

SARI L. 2014 - Technological change in Iberomaurusian culture: The case of Tamar Hat, Rassel and Columnata lithic assemblages (Algeria). Quaternary International, 320, p. 131-142.

SAXON E.C., CLOSE A., CLUZEL C., MORSE V., SHACKELTON N.J. 1974 - Results of recent investigations at Tamar Hat, Libyca, 22, p. 49-91.

SELLA G., ROBOTTI C.A., BIGLIONE V. 1993 - Genetic divergence among three sympatric species of
Mediterranean Patella (Archaeogastropoda). Marine Biology, 115, p. 401-405.

SIDI MAAMAR H. 1987 - Étude de la faune malacologique de l'abri Alain, Oran, Algérie: Réflexion autour d'une escargotière ibéromaurusienne. Paris : Université de Paris I. Mémoire de maîtrise.

STEELE T., E. ALVAREZ-FERNANDEZ E. 2011 - Initial Investigations into the Exploitation of Coastal Resources in North Africa During the Late Pleistocene at Grotte des Contrebandiers, Morocco. In : N. F. Bicho, J.A. Haws, L.A. Davis (Ed.), Trekking the Shore: Changing Coastlines and the Antiquity of Coastal Settlement, New York : Springer, p. 383-403.

STEELE T. et ALVAREZ-FERNANDEZ E. 2012 - Restes de Mollusques marins. In : A. M. El Hajraoui, R. Nespoulet, A. Debénath, H. L. Dibble H.L. (Ed.), La Préhistoire de La Région de Rabat-Témara. Rabat : Villes et sites Archéologiques du Maroc, Vol. III, Rabat : Edition du Ministère de la Culture et de l'Institut National des Sciences de l'Archéologie et du Patrimoine, p. 223-227.

STINER M.C. 1999 - Trends in Paleolithic mollusk exploitation at Riparo Mochi (Balzi Rossi, Italy): food and ornaments from the Aurignacian through Epigravettian. Antiquity, 73, p. 735-754.

STINER M.C. 2003 - Standardization" in Upper Paleolithic Ornaments at the Coastal Sites of Riparo Mochi and Üçagizli Cave. In : J. Zilhão, F. d'Errico (Ed.), The Chronology of the Aurignacian and of the Transitional Technocomplexes: Dating, Stratigraphies, Cultural Implications, Lisbon : Trabalhos de Arqueologia. p. 49-59.

SHINER M.C. et MUNRO N.D. 2011 - On the evolution of diet and landscape during the Upper Paleolithic through Mesolithic at Franchthi Cave (Peloponnese, Greece). Journal of Human Evolution, 60, p. 618-636.

STINER M.C., PEHLEVAN C., SAGIR M. et OZER I. 2002 Zooarchaeological studies at Uçagizli Cave: Preliminary results on Palaeolithic subsistence and shell ornaments. Arkeometri Sonuslari Toplantisi. Ankara, 17, p. 29-36.

STOETZEL E., CAMPMAS E., MICHEL P., BOUGARIANE B., OUCHAOU B., AMANI F., EL HAJRAOUI M.A. et NESPOULET R. 2014 - Context of modern human occupations in North Africa: Contribution of the Témara caves data. Quaternary International, 320, p. $143-161$

TAYLOR V.K., BARTON R.N.E., BELL M., BOUZOUGGAR A., COLLCUTT S., BLACK S., HOGUE J.T. 2011 - The Epipalaeolithic (Iberomaurusian) at Grotte des Pigeons (Taforalt), Morocco: A preliminary study of the land Mollusca. Quaternary International, 244, p. 5-14.

TIXIER J. 1963 - Typologie de l'Epipaléolithique du Maghreb. Paris : Arts et métiers graphiques. 
VANHAEREN M., D'ERRICO F., STRINGER C., JAMES S., L., TODD J. et MIENIS H. 2006 - Middle Paleolithic Shell Beads in Israel and Algeria. Science, 312, p. 1785-1788.

ZILHÃO J., ANGELUCCI D.E., BADAL-GARCIA E., D'ERRICO F., DANIEL F., DAYET L., DOUKA K., HIGHAM T.F.G., MARTINEZ-SANCHEZ M.J., MONTESBERNARDEZ R., MURCIA-MASCAROS S., PEREZ-
SIRVENT C., ROLDAN-GARCIA C., VANHAEREN M., VILLAVERDE V., WOOD R., ZAPATA J. 2010 - Symbolic use of marine shells and mineral pigments by Iberian Neandertals. Proceeding of National Academic Sciences of United States of America, 107, p. 1023-1028.

ZOUGHLAMI J., HARBI-RIAHI M., GRAGUEB A. et CAMPS G. 1985 - Atlas Préhistorique de la Tunisie 23 Gabès, Rome : École française de Rome. 